\title{
Lattice calculus of the morphological slope transform
}

\author{
Henk J.A.M. Heijmans ${ }^{a}$ * , Petros Maragos ${ }^{b, 1}$ \\ ${ }^{a}$ CWI, P.O. Box 94079, 1090 GB Amsterdam. The Netherlands \\ ${ }^{\mathrm{b}}$ School of Electrical and Computer Engineering, Georgia Institute of Technology, Atlanta, GA 30332-0250, USA
}

Received 11 July 1996; revised 28 January 1997

\begin{abstract}
This paper presents a study of the morphological slope transform in the complete lattice framework. It discusses in detail the interrelationships between the slope transform at one hand and the (Young-Fenchel) conjugate and Legendre transform, two well-known concepts from convex analysis, at the other. The operators and transforms of importance here (hull operations, slope transform, support function, polar, gauge, etc.) are complete lattice operators with interesting properties also known from theoretical morphology. For example, the slope transform and its 'inverse' form an adjunction. It is shown that the slope transform for sets (binary signals) coincides with the notion of support function, known from the theory of convex sets. Two applications are considered: the first application concerns an alternative approach to the distance transform. The second application deals with evolution equations for multiscale morphology using the theory of Hamilton--Jacobi equations. (C) 1997 Elsevier Science B.V.
\end{abstract}

\section{Zusammenfassung}

In diesem Beitrag wird eine Untersuchung der morphologischen Steigungstransformation (morphological slope transform) im Rahmen der vollständigen lattice-Theorie präsentiert. Dabei werden die Zusammenhänge zwischen der Steigungstransformation einerseits und der (Young-Fenchel) konjugierten Transformation sowie der Legendre Transformation andererseits, die zwei wohlbekannte Verfahren aus der konvexen Analyse darstellen, diskutiert. Die Operatoren und Transformationen, die hier von Bedeutung sind (hull-Operationen, Steigungstransformation, support-Funktion, polar, gauge, etc.) stellen vollständige lattice-Operatoren mit interessanten Eigenschaften dar, die ebenfalls aus der theoretischen Morphologie bekannt sind. Die Steigungstransformation beispielsweise bildet zusammen mit ihrer Inversen ein adjungiertes Paar. Es wird gezeigt, daß die Steigungstransformation für Mengen (binäre Signale) mit der Kenntnis der support-Funktion einhergeht, die aus der Theorie konvexer Mengen bekannt ist. Es werden zwei Anwendungen betrachtet: die erstc betrifft einen alternativen Ansatz zur Distanz-Transformation. Bei der zweiten geht es um Evolutionsgleichungen in der multiscale-Morphologie unter Ausnutzung der Theorie der Hamilton-Jacobi Gleichungen. (C) 1997 Elsevier Science B.V.

\section{Résumé}

Cet article présente une étude de la transformation de pente morphologique dans un cadre de structure en treillis. Il discute en détail les inter-relations entre la transformation de pente d'un côté et les transformations conjuguée (de Young-Fenchel) et de Legendre, deux concepts bien connus en analyse complexe, de l'autre. Les opérateurs et les transformations d'importance

\footnotetext{
* Corresponding author. Address: CWI, Kruislaan 413, 1098SJ Amsterdam, The Netherlands. Tel.: +31 205924057; fax: +3120592 4199; e-mail: henkh@cwi.nl.

${ }^{1}$ The research work of P. Maragos was supported by the US NSF under Grants MIP-9396301 and MIP-9421677, and by the ARO under Grant DAAH04-96-1-0161.
} 
ici (opérateurs d'enveloppe convexe, transformation de pente, fonction de support, polaire, jauge, etc.) sont des opérateurs de treillis complets avec des propriétés intéressantes connues aussi par le biais de la morphologie théorique. Par exemple, la transformation de pente et son 'inverse' forment une adjonction. Il est montré que la transformation de pente pour des ensembles (signaux binaires) coincide avec la notion de fonction de support, provenant de la théorie des ensembles convexes. Deux applications sont considérées: la première est relative à une approche alternative de la transformation de distance. La deuxième a trait aux équations d'évolution pour la morphologie multi-échelle par le biais de la théorie des équations de Hamilton-Jacobi. (C) 1997 Elsevier Science B.V.

Key'ords: Morphological systems for signal analysis; Adjunctions; Convex sets; Supremal and infimal convolution; Upper and lower slope transform; Lipschitz continuous functions; Upper semi-continuous (u.s.c.) and lower semi-continuous (l.s.c.) functions; Convex functions; Conjugation; Distance transform; Morphological evolution equation

\section{Introduction}

Morphological signal analysis is becoming an important area of nonlinear functional analysis that has found many applications in image processing and nonlinear filtering. The morphological signal operators are parallel or serial interconnections of morphological dilations and erosions, respectively, defined as

$$
\begin{aligned}
& (f \oplus g)(x)=\bigvee_{y \in \mathbb{R}^{\prime}} f(x-y)+g(y), \\
& (f \oplus g)(x)=\bigwedge_{y \in \mathbb{R}^{\prime}} f(x+y)-g(y),
\end{aligned}
$$

where $\bigvee$ denotes supremum and $\bigwedge$ denotes infimum. The theory of deterministic morphological operators is quite rich and has been based on set and lattice theory $[11,19,25,26]$. In spite of their wide applicability, so far their analysis has been done only in the time (or spatial) domain because of lack of transforms which enable us to also describe them in a transform domain. However, recently some nonlinear signal transformations have been introduced in $[7,17,18]$, called 'slope transforms', which endow morphological systems with eigenfunctions and a related transfer function in a slope domain. It turns out that the morphological slope transforms, restricted on the class of concave or convex functions, are closely related to the conjugate functions of convex analysis $[8,13,14,21,22]$.

Therefore, there are many interesting ideas in the overlapping among the areas of morphological systems, slope transforms, and convex analysis. In this paper, we show that an efficient methodology and mathematically elegant framework to study and further advance these interrelationships is lattice theory as applied to mathematical morphology. Thus, although the slope transforms are intended for analysis of morphological systems, they can benefit from the already developed theory of conjugate functions in convex analysis. Thus one of the contributions of this paper is to use convex analysis to enrich the understanding of slope transforms. Further, both areas can benefit from using the framework of complete lattices for studying the signal classes and operations involved. Thus another contribution of the paper is to study slope transforms in the context of complete lattices. Further, a rich class of signals used in morphological image analysis is that of binary signals, which are viewed as indicator functions of sets. A goal of this paper is to study the slope transforms of binary signals, which turn out to be the support functions of the corresponding sets, a concept very frequently used in convex analysis. Finally, in convex analysis, the use of conjugate functions for both multilevel and binary signals is constrained to the cases of convex or concave signals. In this paper we apply slope transforms to arbitrary signals, even if the information in the original signal is not always completely recoverable from its slope transform. Unless stated otherwise, the propositions and corollaries in this paper are new (to the best of our knowledge).

We begin in Section 2 with some basic notions from the theory of morphological signal processing. First, we briefly describe the complete lattice framework of mathematical morphology. Next, we remind the reader of the classical linear theory of signal processing and the corresponding Fourier approach. We show that there exist several analogies between the linear and the morphological approach. The emphasis is laid 
Upon the slope transform which may be considered as the morphological counterpart of the Fourier transform. In Section 3 we study the morphological slope transform within the framework of complete lattices. Since the slope transform is closely related to conjugation, we can use concepts from convex analysis here. Section 4 focuses on the slope transform of the indicator function of a set and its relationships to the Set's support function, again viewed in the context of complete lattices. In Section 5 we discuss some applications of the ideas in this paper. Our first example concerns the distance transform, the second example discusses nonlinear partial differential equations that describe multiscale morphological operations $[6,20,29]$. We show that the resulting PDEs can be reformulated as Hamilton-Jacobi equations which have been thoroughly studied in the literature. We end with some conclusions in Section 6 .

\section{Morphological signal processing}

\section{1. Morphology on complete lattices with appli- cations to convex sets}

A set $\mathscr{L}$ with a partial ordering $\leqslant$ is called a complete lattice if every subset $\mathscr{H} \subseteq \mathscr{L}$ has a supremum (least upper bound) $\bigvee \mathscr{H}$ and infimum (greatest lower bound) $\bigwedge \mathscr{H}$; refer e.g. to [4]. The opposite of $\mathscr{L}$, denoted by $\mathscr{L}^{\prime}$, is the complete lattice with partial ordering $X \leqslant{ }^{\prime} Y$ iff $X \geqslant Y$. A comprehensive discussion of the theory of morphological operators on complete lattices can be found in [11].

Let $\mathscr{L}, \mathscr{U}$ be complete lattices. A pair of operators $(\varepsilon, \delta)$, where $\varepsilon: \mathscr{L} \rightarrow \mathscr{U}$ and $\delta: \mathscr{U} \rightarrow \mathscr{L}$, which obeys

$\delta(Y) \leqslant X \Leftrightarrow Y \leqslant \varepsilon(X), \quad X \in \mathscr{L}, Y \in \mathscr{M}$,

is called an adjunction between $\mathscr{L}$ and $\mathscr{U}$. In that case, $\varepsilon$ and $\delta$ distribute over infima and suprema, respectively,

$$
\begin{aligned}
& \varepsilon\left(\bigwedge_{i \in I} X_{i}\right)=\bigwedge_{i \in I} \varepsilon\left(X_{i}\right), \\
& \delta\left(\bigvee_{j \in J} Y_{j}\right)=\bigvee_{j \in J} \delta\left(Y_{j}\right),
\end{aligned}
$$

for arbitrary collections $\left\{X_{i} \mid i \in I\right\} \subseteq \mathscr{L}$ and $\left\{Y_{j} \mid j \in\right.$ $J\} \subseteq \| \mathscr{U}$. An operator $\varepsilon$ which satisfies (2.2) is called an erosion. An operator $\delta$ which satisfies (2.3) is called a dilation. Erosions and dilations are increasing mappings: a mapping $\psi: \mathscr{L} \rightarrow \mathscr{H}$ is called increasing if $X_{1} \leqslant X_{2}$ implies that $\psi\left(X_{1}\right) \leqslant \psi\left(X_{2}\right)$, for $X_{1}, X_{2} \in \mathscr{L}$. The range of $\psi$ is $\operatorname{Ran}(\psi)=\{\psi(X) \mid X \in \mathscr{L}\}$.

With every erosion $\varepsilon: \mathscr{L} \rightarrow \mathscr{M}$ there corresponds a unique dilation $\delta: \mathscr{H} \rightarrow \mathscr{L}$ such that $(\varepsilon, \delta)$ constitutes an adjunction. Vice versa, with every dilation $\delta: \mathscr{H} \rightarrow \mathscr{L}$ there corresponds a unique erosion $\varepsilon: \mathscr{L} \rightarrow \mathscr{U}$ such that $(\varepsilon, \delta)$ constitutes an adjunction. We say that $\delta$ is the adjoint dilation of $\varepsilon$, and also that $\varepsilon$ is the adjoint erosion of $\delta$.

If $(\varepsilon, \delta)$ is an adjunction between $\mathscr{L}$ and $\mathscr{U}$, then

$\varepsilon \delta \varepsilon=\varepsilon \quad$ and $\quad \delta \varepsilon \delta=\delta$.

Also

$\varepsilon \delta \geqslant i d_{. / l}$ and $\delta \varepsilon \leqslant i d_{\varphi}$

here $i d_{\mathscr{P}}, i d_{\text {, }}$ represent the identity mappings on $\mathscr{L}$ and $\mathscr{l l}$, respectively.

An operator $\psi: \mathscr{L} \rightarrow \mathscr{L}$ is called an opening if it is increasing, idempotent (i.e., $\psi^{2}=\psi$ ), and antiextensive (i.e., $\psi \leqslant i d$ ). It is called a closing if it is increasing, idempotent, and extensive (i.e., $\psi \geqslant i d$ ). If $(\varepsilon, \delta)$ is an adjunction between $\mathscr{L}$ and $\mathscr{M}$, then $\delta \varepsilon$ is an opening on $\mathscr{L}$ and $\varepsilon \delta$ is a closing on $\mathscr{M}$. Openings will be denoted by $\alpha$ and closings by $\beta$.

The following result will be used later on in this paper.

Proposition 2.1. Let $(\varepsilon, \delta)$ be an adjunction between $\mathscr{L}$ and $\mathscr{l l}$.

(a) If $X_{i} \in \operatorname{Ran}(\delta)$ for $i \in I$, then

$$
\varepsilon\left(\bigvee_{i \in I} X_{i}\right)=\varepsilon \delta\left(\bigvee_{i \in I} \varepsilon\left(X_{i}\right)\right)
$$

(b) If $Y_{j} \in \operatorname{Ran}(\varepsilon)$ for $j \in J$, then

$$
\delta\left(\bigwedge_{j \in J} Y_{j}\right)=\delta \varepsilon\left(\bigwedge_{j \in J} \delta\left(Y_{j}\right)\right) .
$$

Proof. We prove only (a), for then (b) follows by duality. Let $X_{i}=\delta\left(Y_{i}\right)$, then, using that $\delta \varepsilon \delta=\delta$, 
we get

$$
\begin{aligned}
\varepsilon \delta\left(\bigvee_{i \in I} \varepsilon\left(X_{i}\right)\right) & =\varepsilon \delta\left(\bigvee_{i \in I} \varepsilon \delta\left(Y_{i}\right)\right)=\varepsilon\left(\bigvee_{i \in I} \delta \varepsilon \delta\left(Y_{i}\right)\right) \\
& =\varepsilon\left(\bigvee_{i \in I} \delta\left(Y_{i}\right)\right)=\varepsilon\left(\bigvee_{i \in I} X_{i}\right)
\end{aligned}
$$

This concludes the proof.

Remark 2.2. This result can be restated as follows. The subset $\mathscr{L}^{\prime}=\operatorname{Ran}(\delta)$ of $\mathscr{L}$ is a complete lattice with the same supremum as $\mathscr{L}$ but with infimum $\delta \varepsilon\left(\bigvee_{j \in I} X_{i}\right)$. The subset $\mathscr{U}^{\prime}=\operatorname{Ran}(\varepsilon)$ of $\mathscr{U}$ is also a complete lattice with the same infimum as $\mathscr{U}$ but with supremum $\varepsilon \delta\left(\bigvee_{j \in J} Y_{j}\right)$. The pair $(\varepsilon, \delta)$ yields an adjunction between $\mathscr{L}^{\prime}$ and $\mathscr{U}^{\prime}$, and for this restriction, $\varepsilon$ and $\delta$ are each other's inverses.

The invariance domain of an operator $\psi: \mathscr{L} \rightarrow \mathscr{L}$ is defined by

$\operatorname{Inv}(\psi)=\{X \in \mathscr{L} \mid \psi(X)=X\}$

The invariance domain of an opening (respectively closing) is closed under the formation of suprema (respectively infima), that is, if $X_{i} \in \operatorname{Inv}(\psi)$ for $i \in I$, then $\bigvee_{i \in I} X_{i}$ (respectively $\bigwedge_{i \in I} X_{i}$ ) lies in $\operatorname{Inv}(\psi)$ as well. Conversely, if $\mathscr{H} \subseteq \mathscr{L}$ is closed under suprema, then there is a unique opening $\alpha$ on $\mathscr{L}$ with $\operatorname{Inv}(\alpha)=\mathscr{H}$. Dually, if $\mathscr{H} \subseteq \mathscr{L}$ is closed under infima, then there exists a unique closing $\beta$ on $\mathscr{L}$ with $\operatorname{Inv}(\beta)=\mathscr{H}$. Refer to [11] for a proof of these results.

\section{Proposition 2.3.}

(a) Let $\alpha, \alpha^{\prime}$ be openings on $\mathscr{L}$ such that $\alpha^{\prime} \alpha \alpha^{\prime}=\alpha \alpha^{\prime}$, then $\alpha \alpha^{\prime}$ is an opening with invariance domain $\operatorname{Inv}(\alpha) \cap \operatorname{Inv}\left(\alpha^{\prime}\right)$.

(b) Let $\beta, \beta^{\prime}$ be closings on $\mathscr{L}$ such that $\beta^{\prime} \beta \beta^{\prime}=\beta \beta^{\prime}$, then $\beta \beta^{\prime}$ is a closing with invariance domain $\operatorname{Inv}(\beta) \cup \operatorname{Inv}\left(\beta^{\prime}\right)$.

Proof. To see that $\alpha \alpha^{\prime}$ is an opening, we only have to show that $\alpha \alpha^{\prime}$ is idempotent. But this is obvious since $\alpha \alpha^{\prime} \alpha \alpha^{\prime}=\alpha \alpha \alpha^{\prime}=\alpha \alpha^{\prime}$. Furthermore, one sees immediately that $\alpha \alpha^{\prime}=\alpha^{\prime} \alpha \alpha^{\prime}$ maps into $\operatorname{Inv}(\alpha) \cap \operatorname{Inv}\left(\alpha^{\prime}\right)$. On the other hand, if $X \in \operatorname{Inv}(\alpha) \cap \operatorname{Inv}\left(\alpha^{\prime}\right)$, then $X=$ $\alpha(Y)=\alpha^{\prime}\left(Y^{\prime}\right)$ for some $Y, Y^{\prime} \in \mathscr{L}$. Thus, $\alpha \alpha^{\prime}(X)=$ $\alpha \alpha^{\prime} \alpha^{\prime}\left(Y^{\prime}\right)=\alpha \alpha^{\prime}\left(Y^{\prime}\right)=\alpha(X)=\alpha \alpha(Y)=\alpha(Y)=X$.

This proves the result.

We illustrate these abstract concepts by means of some concrete examples. Denote by $\mathscr{P}\left(\mathbb{R}^{d}\right)$ the set of all subsets of $\mathbb{R}^{d}$; the empty set will be denoted by $\emptyset$. Then $\mathscr{P}\left(\mathbb{R}^{d}\right)$ is a complete lattice if we take set inclusion as partial ordering. Supremum and infimum are given by set union and intersection, respectively.

Recall that Minkowski sum and difference of two sets $X, A \subseteq \mathbb{R}^{d}$ are defined as

$X \oplus A=\{x+a \mid x \in X, a \in A\}=\bigcup_{a \in A} X_{a}$,

$X \ominus A=\bigcap_{a \in A} X_{-a}$.

The pair $\left(\varepsilon_{A}, \delta_{A}\right)$, where $\delta_{A}(X)=X \oplus A$ and $\varepsilon_{A}(X)=X \ominus A$, defines an adjunction on $\mathscr{P}\left(\mathbb{R}^{d}\right)$. The set $A$ is called structuring element.

The mapping $X \mapsto \operatorname{int}(X)$ which maps a set $X$ to its interior is an opening. Dually, the mapping $\beta_{\mathrm{c}}$ given by $\beta_{\mathrm{c}}(X)=\bar{X}$, where $\bar{X}$ is the closure of $X$, is a closing.

Recall that a set $X \subseteq \mathbb{R}^{d}$ is convex if $r x+(1-$ $r) y \in X$ for $x, y \in X$ and $0 \leqslant r \leqslant 1$. A set $X$ is called a cone if $r x \in X$ for $x \in X$ and $r \geqslant 0$. A cone which is convex is called a convex cone. See Fig. 1 for some illustrations.

The collection of convex sets in $\mathbb{R}^{d}$ is denoted by $\mathscr{P}_{\wedge}\left(\mathbb{R}^{d}\right)$. This is a complete lattice under the inclusion relation with set intersection as infimum, but with a different supremum, an expression for which is given below. If $X, Y \subseteq \mathbb{R}^{d}$ then $X \oplus Y$ is convex, too. The convex hull $\operatorname{co}(X)$ of a set $X$ is the intersection of all convex sets which contain $X$. Since an intersection of convex sets is convex, $\operatorname{co}(X)$ is a convex set, the smallest convex set which contains $X$. Now the supremum of the collection $\left\{X_{i} \mid i \in I\right\}$ in $\mathscr{P}_{\wedge}\left(\mathbb{R}^{d}\right)$ is given by $\operatorname{co}\left(\bigcup_{i \in I} X_{i}\right)$. The map $\beta_{\wedge}$ on $\mathscr{P}\left(\mathbb{R}^{d}\right)$ given by $\beta_{\wedge}(X)=\operatorname{co}(X)$ is a closing with invariance domain $\mathscr{P}_{\wedge}\left(\mathbb{R}^{d}\right)$.

It is a well-known fact [28] that the closure of a convex set is convex. In operator notation,

$\beta_{\wedge} \beta_{\mathrm{c}} \beta_{\wedge}=\beta_{\mathrm{c}} \beta_{\wedge}$

Now Proposition 2.3(b) gives that $\beta_{\mathrm{c}} \beta_{\wedge}$ is a closing with invariance domain $\operatorname{Inv}\left(\beta_{\wedge}\right) \cap \operatorname{Inv}\left(\beta_{\mathrm{c}}\right)$, the closed 


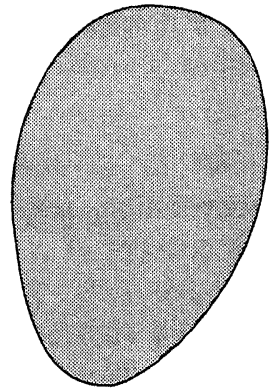

convex set

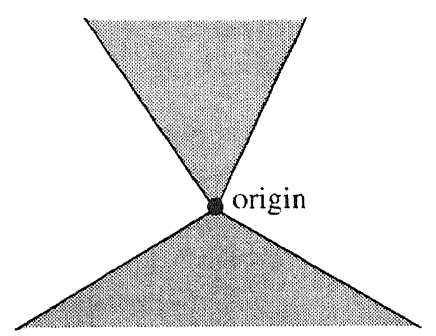

cone

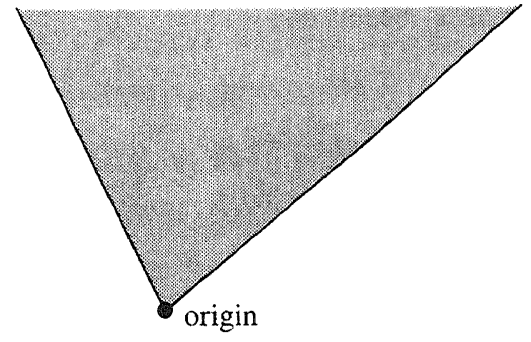

convex cone

Fig. 1. From left to right: a convex set, a cone, and a convex cone.

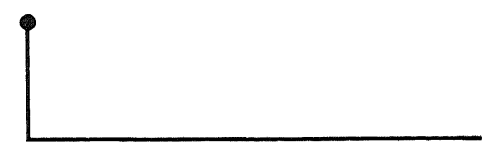

$\mathrm{X}$

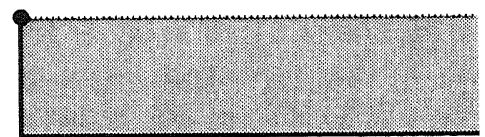

$\operatorname{co}(\mathrm{X})$

Fig. 2. A closed set $X$ for which $\operatorname{co}(X)$ is not closed.

convex sets. The set $\beta_{\mathrm{c}} \beta_{\wedge}(X)=\overline{\operatorname{co}(X)}$ is called the closed convex hull of $X$, also sometimes denoted by $\overline{\mathrm{co}}(X)$.

The example in Fig. 2 shows that the convex hull of a closed set need not be closed, i.e.,

$\beta_{\mathrm{c}} \beta_{\wedge} \beta_{\mathrm{c}} \neq \beta_{\wedge} \beta_{\mathrm{c}}$

The mapping $\delta: \mathscr{P}\left(\mathbb{R}^{d}\right) \rightarrow \mathscr{P}\left(\mathbb{R}^{d}\right)$ given by

$$
\delta(Y)=\bigcup_{r \geqslant 0} r Y
$$

is a dilation. The corresponding erosion is given by

$$
\varepsilon(X)= \begin{cases}\emptyset, & 0 \notin X, \\ \bigcap_{r>0} r X, & 0 \in X .\end{cases}
$$

Both $\varepsilon(X)$ and $\delta(X)$ are cones, for every $X \subseteq \mathbb{R}^{d}$. The set $\varepsilon(X)$ is called asymptotic cone or recession cone if $X$ is convex [13, Section III.2.2]. Note that $\delta$ is also a closing, and, dually, that $\varepsilon$ is an opening.

\subsection{Linear signal processing and Fourier analysis}

A signal operator $\Psi: f \mapsto \Psi(f)$, defined on the space of complex-valued signals with domain $\mathbb{R}^{d}$, is called a linear shift-invariant ( $L S I)$ system if $\Psi$ obeys the linear superposition principle, i.e.,

$\Psi\left(\sum_{i} c_{i} f_{i}\right)=\sum_{i} c_{i} \Psi\left(f_{i}\right)$,

where $\left\{f_{i}\right\}$ is a finite signal collection and $c_{i}$ are constants, and if $\Psi$ is horizontally shift-invariant:

$\Psi\left(f_{y}\right)=[\Psi(f)]_{y}$,

where $f_{y}$ denotes the horizontal translate of the function $f$ over the vector $y$, i.e.,

$f_{y}(x):=f(x-y)$.

The output from $\Psi$ can be found via the linear convolution

$\Psi(f)(x)=(f * h)(x):=\int_{\mathbb{R}^{d}} f(y) h(x-y) \mathrm{d} y$

of the input signal $f(x)$ and the impulse response $h(x)$, which is the system's output due to a Dirac delta 
input. The exponential signals $\exp (j\langle x,(1)\rangle)$ are eigenfunctions of $\Psi$, because

$\Psi(\exp (j\langle x,(\omega)\rangle))=H(\omega) \exp (j\langle x,(\omega\rangle)$,

where $\langle x,(0\rangle)$ denotes the inner product of the vectors $x=\left(x_{1}, \ldots, x_{d}\right)$ and $(\omega)=\left(\omega_{1}, \ldots, \omega_{d}\right)$ in $\mathbb{R}^{d}$ :

$\langle x, \omega\rangle:=\sum_{i=1}^{d} x_{i} \omega_{i}$

Note that we use the 'loose notation' $\Psi(\exp (j\langle x, \omega\rangle))$ instead of $\Psi(\exp (j\langle\cdot,(i)\rangle))$.

The eigenvalue $H(\omega)$, called the system's frequency response, is the Fourier transform of $h(x)$ :

$H(\omega)=\int_{\mathbb{R}^{\prime}} h(x) \exp (-j\langle x, \omega\rangle) \mathrm{d} x$.

The frequency response provides a simple way to find the system's output when the input is a weighted sum of sinusoids, because the output will also be a weighted sum of sinusoids with same frequencies and with amplitudes and phase offsets determined by $H(\omega)$. In addition, the frequency response may often be a simpler description of the system, especially in the case of a frequency-selective (e.g., low pass or band pass) filter, because signal convolution becomes multiplication of their Fourier transforms; thus,

$g=f * h \Leftrightarrow G=F H$,

where $F, G$ are the Fourier transforms of $f, g$.

\subsection{Morphological systems and supremal/infimal convolution}

In convex analysis and optimization $[2,13$, $14,21,22,30]$, the nonlinear signal operation $\oplus$ given by (1.1) is usually called supremal convolution. A dual operation is the so-called infimal convolution given by

$(f \square g)(x)=\bigwedge_{y \in \mathbb{R}^{d}} f(x-y)+g(y)$.

Note that $\square$ is closely related to the morphological erosion $\ominus$, given by (1.2), because

$f \ominus g=f \square(-\breve{g})$, where $\breve{g}$ is the reflection of $g$ given by

$\check{g}(x)=g(-x)$.

Henceforth, we shall refer to $\oplus$ and $\square$ as the supremal and infimal convolution, respectively, to distinguish them from the concept of a dilation and erosion operator on a lattice.

A mapping $\Delta$ which sends a signal $f$ to a transformed signal $\Delta(f)$ is called a dilation translationinvariant $(D T I)$ system if it is a dilation, i.e., $\Delta\left(\bigvee_{i} f_{i}\right)=\bigvee_{i} \Delta\left(f_{i}\right)$, and if it is translation-invariant, i.e., $\Delta\left(f_{y}+c\right)=\Delta(f)_{y}+c$ for any shift $y$ and any real constant $c$. It is easy to verify that a system is DTI if it is horizontally shift-invariant and obeys the morphological supremum superposition principle

$\Delta\left[\bigvee_{i \in I} f_{i}(x)+c_{i}\right]=\bigvee_{i \in I}\left[\Delta\left(f_{i}\right)(x)+c_{i}\right]$,

where $\left\{f_{i}\right\}$ is any signal collection and $c_{i} \in \mathbb{R}$.

Many important aspects of a DTI system can be determined in the time or spatial domain solely from knowledge of its output signal due to an elementary input signal, the morphological lower impulse $q_{\wedge}$ given by

$q \wedge(x):= \begin{cases}0, & x=0 \\ -\infty, & x \neq 0 .\end{cases}$

The corresponding output of the DTI system $\Delta$ when the input is the lower impulse is henceforth defined as its lower impulse response

$g:=\Delta(q \wedge)$

This uniquely characterizes a DTI system in the time domain, because any DTI system is equivalent to a supremal convolution (also called 'morphological dilation') by its lower impulse response:

$\Delta(f)=f \oplus g$.

Similarly, a signal operator $\mathscr{E}: f \mapsto \mathscr{E}(f)$ is called an erosion translation invariant (ETI) system if it is horizontally shift-invariant and obeys the morphological infimum superposition principle

$\mathscr{E}\left[\bigwedge_{i \in I} f_{i}(x)+c_{i}\right]=\bigwedge_{i \in I}\left[\mathscr{E}\left(f_{i}\right)(x)+c_{i}\right]$ 
where $c_{i} \in \mathbb{R}$. If we define the upper impulse response $h$ of an ETI systems $\mathscr{E}$ as its response

$h:=\mathscr{E}(q \vee)$

to the upper impulse

$q \vee(x):= \begin{cases}0, & x=0, \\ +\infty, & x \neq 0,\end{cases}$

then it follows that

$\mathscr{E}(f)=f \square h$

When the ETI and DTI systems are related via an adjunction, then there is also a close relationship between their impulse responses. Namely, let $\mathscr{E}$ be an ETI system, and let $\Delta$ be its adjoint dilation. It is easy to show that $\Delta$ is a DTI system [11], and therefore $\Delta(f)=f \oplus g$, where $g$ is the lower impulse response. Now

$\mathscr{E}(f)=f \ominus g$

Note that (2.9) and (2.8) become identical if one puts $h=-\check{g}$.

Remark 2.4. Since we are dealing with functions mapping into the extended reals, we have to provide some rules for addition and multiplication of such numbers; see also [13, Appendix 2]. Such rules have to be in correspondence with certain properties of dilations and erosions on the complete lattice $\overline{\mathbb{R}}$. From the fact that a dilation $d: \overline{\mathbb{R}} \rightarrow \overline{\mathbb{R}}$ satisfies $d(-\infty)=-\infty$ (see $[11$, Chapter 11]) we get that $-\infty+(+\infty)=-\infty$ if it occurs in an expression like $f \oplus g$. However, in $f \ominus g$ we have to put $-\infty-(-\infty)=+\infty$. In many cases, however, $g$ will be finite everywhere. Finally, we will put $0 \cdot-\infty=0 \cdot+\infty=0$.

\subsection{Upper and lower slope transform}

To analyze morphological systems in a transform domain, the following two signal transforms were introduced in $[17,18]$. Given a signal $f$, its upper slope transform is defined as

$\mathscr{S}_{\vee}(f)(v):=\bigvee_{x \in \mathbb{R}^{d}} f(x)-\langle x, v\rangle, \quad v \in \mathbb{R}^{d}$, and its lower slope transform is

$$
\mathscr{T}_{\wedge}(f)(v):=\bigwedge_{x \in \mathbb{R}^{\prime l}} f(x)-\langle x, v\rangle, \quad v \in \mathbb{R}^{d} .
$$

These slope transforms provide information about the slope content of signals and a description of morphological systems in a 'slope domain', with functionality similar to the use of Fourier or Laplace transforms in linear systems. Specifically, the hyperplanes $x \mapsto\langle x, v\rangle+b$ (or lines $x \mapsto v x+b$ for onedimensional systems) are eigenfunctions of any DTI system $\Delta$ because

$\Delta(\langle x, v\rangle+b)=\langle x, v\rangle+b+g^{\vee}(v)$,

if $\Delta$ is given by $(2.7)$ and $y^{\vee}=\mathscr{S}(g)$. We call $g^{\vee}$ the upper slope response of the DTI system $\Delta$. It measures the amount of shift in the intercept of the input lines with slope $v$. It is also conceptually similar to the frequency response of LTI systems which is their multiplicative eigenvalue for input exponentials, whereas $g^{\vee}(v)$ is the additive eigenvalue of DTI systems for input lines with slope $v$. Further, as the frequency response of an LTI system is equal to the Fourier transform of the system's impulse response, in a similar way the slope response of a DTI system is the upper slope transform of the system's lower impulse response.

Perhaps the most important property of Fourier transforms in analyzing LTI systems is their ability to map a linear convolution of signals in the time/spatial domain to multiplication of their Fourier transforms. Similarly, supremal convolution of two signals becomes addition of their upper slope transforms:

$\mathscr{S}_{\vee}(f \oplus g)=f^{\vee}+g^{\vee}$.

Similar ideas apply to ETI systems. Specifically, the above hyperplanes are also eigenfunctions of any ETI system given by (2.8):

$\mathscr{E}(\langle x, v\rangle+b)=\langle x, v\rangle+b+h^{\wedge}(v)$,

where $h^{\wedge}=\mathscr{S}_{\wedge}(h)$. In the special case where the ETI system $\mathscr{E}$ and the DTI system $\Delta$ form an adjunction, their slope responses are closely related since

$h^{\wedge}(v)=-g^{\vee}(v)$.

In general, we note that

$\mathscr{S}_{\vee}(f)(v)=-\mathscr{S}_{\wedge}(-f)(-v)$. 


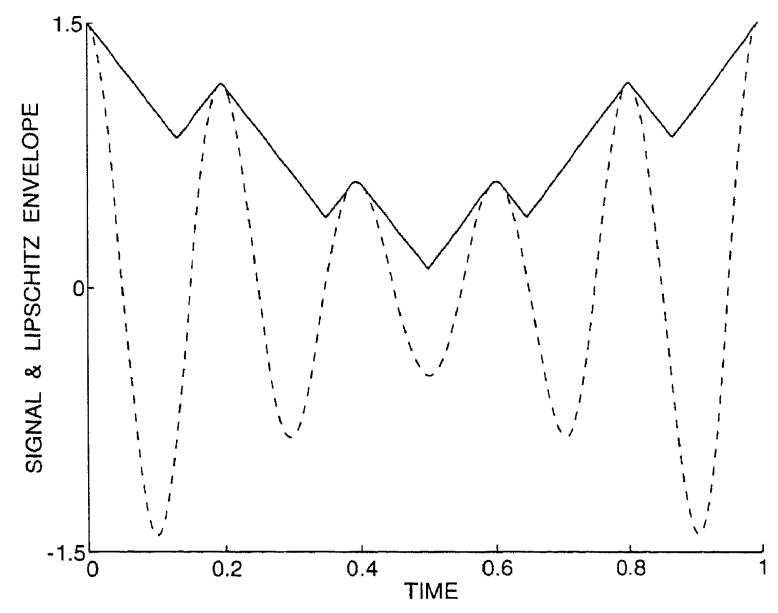

Fig. 3. Slope-limiting (i.e., Lipschitz regularization) of a function via its supremal convolution with a cone. The dashed line shows the original signal $f(x)=[1+0.5 \cos (2 \pi x)] \cos (10 \pi x), x \in[0,1]$. The solid line is the supremal convolution of $f$ with $K_{a}(x)=-a|x|$ where $a=-5$.

From (2.10)-(2.13), one observes that the closing $\mathscr{E} \Delta$ and the opening $\Delta \mathscr{E}$ leave the hyperplanes $x \mapsto\langle x, v\rangle+$ $b$ invariant. For further properties as well as graphical illustrations, the reader may refer to Section 3 .

\subsection{Slope-limited functions, Lipschitz regulariza- tion, and slope filtering}

Define, for every $a>0$, the concave conical function $K_{a}(x)=-a\|x\|$.

Here $\|x\|$ denotes the length (or Euclidean norm) of the vector $x$, i.e., $\|x\|=\left(\left|x_{1}\right|^{2}+\cdots+\left|x_{d}\right|^{2}\right)^{1 / 2}$. Define, for a function $f: \mathbb{R}^{d} \rightarrow \mathbb{R}$ the mapping

$\Psi_{a}(f)=f \oplus K_{a}$,

depending on the slope parameter $a$. See Fig. 3 for an illustration of a one-dimensional signal $f$ and its transformation $\Psi_{a}(f)$.

The family $\left\{\Psi_{a} \mid a>0\right\}$ has the semigroup property

$$
\Psi_{b} \Psi_{a}=\Psi_{a} \Psi_{b}=\Psi_{a \wedge b}, \quad a, b>0
$$

This follows easily from $K_{a} \oplus K_{b}=K_{a \wedge b}$. This latter identity becomes obvious by using (2.11) and the expression for $K_{a}^{\vee}$ given below.
Every $\Psi_{a}$ is a dilation and a closing at the same time. Since the slope transform of the conical function assumes only 0 and $+\infty$ values,

$K_{a}^{\vee}(v)= \begin{cases}0, & |v| \leqslant a, \\ +\infty, & |v|>a,\end{cases}$

and supremal convolution becomes addition in the slope domain,

$\mathscr{S}_{\vee}\left(\Psi_{a}(f)\right)=f^{\vee}+K_{a}^{\vee}$,

it follows that $\Psi_{a}(f)^{\vee}(v)=+\infty$ for $|v|>a$. Hence it is 'upper slope-limited', where we call a function $f: \mathbb{R} \rightarrow \overline{\mathbb{R}}$ upper slope-limited if there exists some $a>0$ such that $f^{\vee}(v)=+\infty$ for $|v|>a$. The constant $a$ may then be called the upper slope bandwidth of $f$. The above discussion implies that a function $f$ becomes upper slope-limited with bandwidth $a$ after its supremal convolution with the cone $K_{a}$.

Slope-limited functions are related to Lipschitz continuous functions. Consider functions $f: \mathbb{R}^{d} \rightarrow \overline{\mathbb{R}}$. Recall that $f$ is Lipschitz if there exists a constant $c>0$ such that $|f(x)-f(y)| \leqslant c\|x-y\|$, for $x, y \in \mathbb{R}^{d}$. If a function $f$ is Lipschitz continuous with constant $a$, then $\Psi_{a}(f)=f$, hence $f$ is an upper slope-limited function with bandwidth $a$. The converse is not true in general. For example, the quadratic function $f(x)=\|x\|^{2}$ has upper slope transform which is identically $+\infty$, but $f$ is obviously not Lipschitz continuous. However, as shown in [22, p.116], a proper concave function $f$ is Lipschitz with constant $a$ if and only if it is upper slope-limited with bandwidth $a$. Note the similarity with Fourier analysis where a real-valued function $f(x)$ is band-limited (i.e., frequency-limited) with bandwidth $\omega_{0}$ if its Fourier transform is zero for frequencies $|\omega|>\omega_{0}$. If a function is not originally band-limited, it can become so by linearly convolving it with the sine-function $h(x)=\sin \left(\omega_{0} x\right) / \pi x$. Band-limiting causes a regularization to the original function because it eliminates higher frequencies in the input. Thus, slope-limiting can be seen as a 'Lipschitz regularization'; see also [14, Example 3.4.4].

Frequency band-limiting can be seen as frequencyselective filtering in the frequency domain, where the input signal components whose frequencies are within the filter's pass band pass unchanged, whereas other frequency components are rejected. Similarly, slope 
transforms have been used for designing and analyzing DTI or ETI systems that act as slope-selective filters $[17,18]$. For example, the above supremal convolutions with the conical functions $K_{a}$ can be seen as symmetric low-pass slope-selective filtering because if the input signal $f$ contains any segments with slopes absolutely greater than $a$, they will be rejected, whereas slopes absolutely smaller than $a$ will pass unchanged. For a more general (asymmetric bandpass) slope filter, imagine a one-dimensional DTI system that passes all line components with slopes in the band $\left[v_{1}, v_{2}\right]$ unchanged, and rejects all the rest. Then its slope response would be

$g^{\vee}(v)= \begin{cases}0, & v_{1} \leqslant v \leqslant v_{2} \\ +\infty, & \text { else }\end{cases}$

This is a general ideal-cutoff slope band-pass filter. In the spatial domain, it acts as a supremal convolution by the impulse response

$g(x)= \begin{cases}v_{1} x, & x \geqslant 0 \\ v_{2} x, & x \leqslant 0 .\end{cases}$

The points on and below the graph of this function $g$, the so-called umbra (see Section 3.1), form a concave cone. Such a dilation by an infinite cone produces upper envelopes of the input signal, as shown in Fig. 3 for the symmetric case $v_{2}=-v_{1}=a>0$.

Lipschitz functions, and more generally, equicontinuous functions play an important role in morphological sampling schemes for grey-scale images [27]. Readers who are interested in an abstract treatment of Lipschitz functions in the complete lattice framework for morphology should refer to [23]. In this paper one find various results related to the ones above.

\section{The slope transform}

\subsection{Complete lattice theory for functions}

We denote by Fun $\left(\mathbb{R}^{d}\right)$ the functions mapping $\mathbb{R}^{d}$ into the extended reals $\overline{\mathbb{R}}=\mathbb{R} \cup\{-\infty,+\infty\}$. It is evident that this defines a complete lattice under the partial ordering given by pointwise inequality: $f_{1} \leqslant f_{2}$ if $f_{1}(x) \leqslant f_{2}(x)$ for every $x \in \mathbb{R}^{d}$. By $f \equiv c$, where $c \in \mathbb{\mathbb { R }}$, we mean that $f(x)=c$, for every $x \in \mathbb{R}^{d}$. The function which equals $c$ everywhere is denoted by ' $\equiv c$ '.
The upper and lower domain of a function $f$ are defined as

$\operatorname{dom}_{\vee}(f)=\left\{x \in \mathbb{R}^{d} \mid f(x)>-\infty\right\}$,

$\operatorname{dom}_{\wedge}(f)=\left\{x \in \mathbb{R}^{d} \mid f(x)<+\infty\right\}$,

respectively. It is easy to show that

$\operatorname{dom}_{\vee}\left(\bigvee_{i \in I} f_{i}\right)=\bigcup_{i \in I} \operatorname{dom}_{\vee}\left(f_{i}\right)$,

$\operatorname{dom}_{\wedge}\left(\bigwedge_{i \in I} f_{i}\right)=\bigcap_{i \in I} \operatorname{dom}_{\wedge}\left(f_{i}\right)$,

for an arbitrary collection $\left\{f_{i} \mid i \in I\right\}$ in $\operatorname{Fun}\left(\mathbb{R}^{d}\right)$. In other words, $\operatorname{dom}_{\vee}(\cdot)$ (respectively $\left.\operatorname{dom}_{\wedge}(\cdot)\right)$ is a dilation (respectively erosion) from Fun $\left(\mathbb{R}^{d}\right)$ into $\mathscr{P}\left(\mathbb{R}^{d}\right)$. Furthermore, we define the epigraph and hypograph of a function as

$U_{\vee}(f)=\left\{(x, t) \in \mathbb{R}^{d} \times \mathbb{R} \mid t \geqslant f^{\prime}(x)\right\}$,

$U_{\wedge}(f)=\left\{(x, t) \in \mathbb{R}^{d} \times \mathbb{R} \mid t \leqslant f(x)\right\}$.

In mathematical morphology, the set $U_{\wedge}(f)$ is usually called the umbra of $f$. Note that $U_{\vee}$ (respectively $U_{\wedge}$ ) defines a dilation (respectively erosion) from $\operatorname{Fun}\left(\mathbb{R}^{d}\right)$ into $\mathscr{P}\left(\mathbb{R}^{d} \times \mathbb{R}\right)$. For an illustration of these concepts we refer to Fig. 4.

A function of the form $x \mapsto\langle x, v\rangle+b$, where $v \in \mathbb{R}^{d}$ and $b \in \overline{\mathbb{R}}$ is called an affine function. If $b= \pm x$ then this function is identically $\pm \infty$, and it is called a degenerate affine function.

Definition 3.1. Let $f$ be an element of Fun $\left(\mathbb{R}^{d}\right)$.

(a) The function $f$ is u.s.c. (upper semi-continuous) if, for every $t \in \overline{\mathbb{R}}$ and $x \in \mathbb{R}^{d}, f(x)<t$ implies that $f(y)<t$, for every $y$ in some neighborhood of $x$.

(b) The function $f$ is l.s.c. (lower semi-continuous) if, for every $t \in \overline{\mathbb{R}}$ and $x \in \mathbb{R}^{d}, f(x)>t$ implies that $f(y)>t$, for every $y$ in some neighborhood of $x$.

The collections of u.s.c. and 1.s.c. functions are denoted by $\operatorname{Fun}_{u}\left(\mathbb{R}^{d}\right)$ and $\operatorname{Fun}_{l}\left(\mathbb{R}^{d}\right)$, respectively. The following result is well-known; see e.g. [22, Section 7]. 


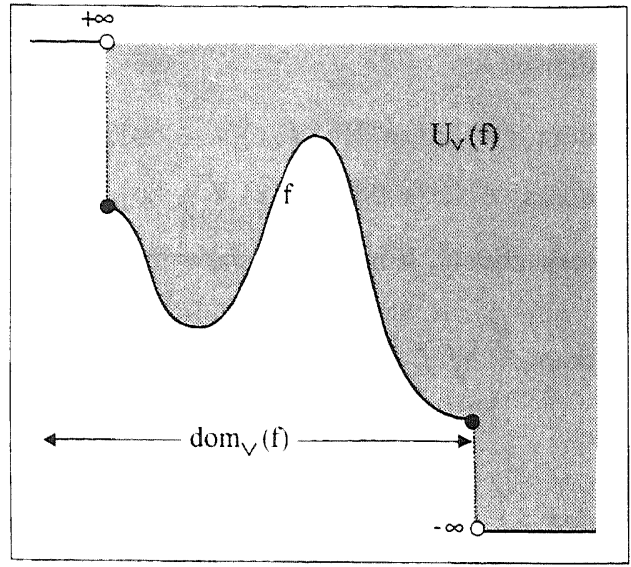

(a)

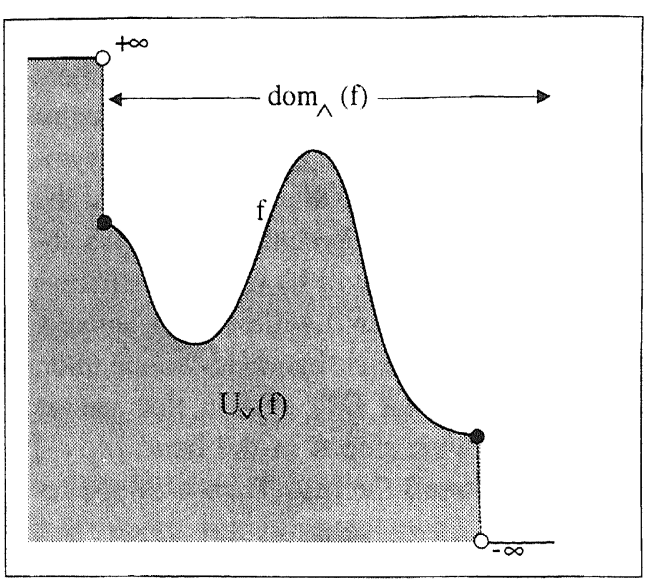

(b)

Fig. 4. (a) Upper domain and epigraph of a function; (b) lower domain and hypograph (or umbra) of a function.

\section{Proposition 3.2.}

(a) A function $f$ is u.s.c. iff its hypograph $U_{\wedge}(f)$ is closed.

(b) A function $f$ is l.s.c. iff its epigraph $U_{\vee}(f)$ is closed.

The infimum of an arbitrary collection of u.s.c. functions is u.s.c. One can use a direct argument to prove this, but one can also exploit the fact that $U_{\wedge}$ is an erosion. Assume that $f_{i}$ is u.s.c. for every $i$ in some index set $I$, then $U_{\wedge}\left(\bigwedge_{i \in I} f_{i}\right)=\bigcap_{i \in I} U_{\wedge}\left(f_{i}\right)$, which, being an intersection of closed sets, is closed. Now Proposition 3.2(a) yields that $\bigwedge_{i \in l} f_{i}$ is u.s.c. Dually, it follows that the supremum of a given collection of 1.s.c. functions is l.s.c.

Let $f$ be an arbitrary function. Define the upper closed hull $\beta_{\mathrm{u}}(f)=\bar{f}$ of $f$ as the infimum of all u.s.c. functions which lie above $f$. Then $\bar{f}$ is u.s.c.; it is the smallest u.s.c. function above $f$. One can easily show that $\bar{f}(x)=\limsup _{y \rightarrow x} f(y)$, and that

$U_{\wedge}(\bar{f})=\overline{U_{\wedge}(f)}$.

Dually, we define the lower closed hull $\alpha_{f}(f)=\underline{f}$ of $f$ as the supremum of all 1.s.c. functions below $f$. The function $f$ is the largest l.s.c. function below $f$, $f(x)=\lim \inf _{y \rightarrow x} f(y)$, and

$U_{\vee}(f)=\overline{U_{\vee}(f)}$.

Now the following result is obvious.
Proposition 3.3.

(a) The mapping $\beta_{\mathrm{u}}$ defines a closing on $\operatorname{Fun}\left(\mathbb{R}^{d}\right)$ with invariance domain $\operatorname{Fun}_{u}\left(\mathbb{R}^{d}\right)$.

(b) The mapping $\alpha$, defines an opening on Fun $\left(\mathbb{R}^{d}\right)$ with invariance domain $\mathrm{Fun}_{l}\left(\mathbb{R}^{d}\right)$.

The next result is a straightforward consequence of the previous observations; cf. [11, Theorem 10.13].

Proposition 3.4.

(a) The set $\operatorname{Fun}_{u}\left(\mathbb{R}^{d}\right)$ is a complete lattice under the pointwise partial ordering with the pointwise infimum $\bigwedge_{i \in I} f_{i}$, and with supremum given by $\beta_{\mathrm{u}}\left(\bigvee_{i \in I} f_{i}\right)$.

(b) The set $\operatorname{Fun}_{l}\left(\mathbb{R}^{d}\right)$ is a complete lattice under the pointwise partial ordering with the pointwise supremum $\bigvee_{i \in I} f_{i}$, and with infimum given by $\alpha_{f}\left(\bigwedge_{i \in I} f_{i}\right)$.

\subsection{Convex and concave functions}

A function $f$ is concave if its hypograph $U_{\wedge}(f)$ is convex, i.e.,

$f(r x+(1-r) y) \geqslant r f(x)+(1-r) f(y)$,

for $x, y \in \mathbb{R}^{d}$ such that $f(x), f(y)>-\infty$ and $0 \leqslant r \leqslant 1$. The function $f$ is convex if its epigraph $U_{\vee}(f)$ is convex, i.e.,

$f(r x+(1-r) y) \leqslant r f(x)+(1-r) f(y)$, 


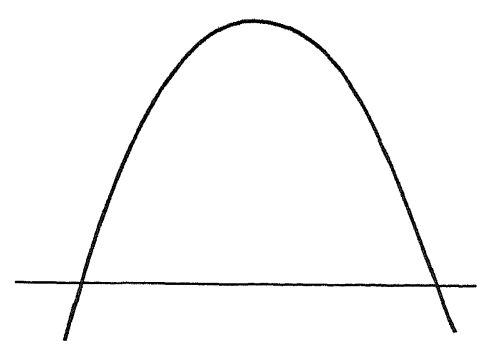

concave function

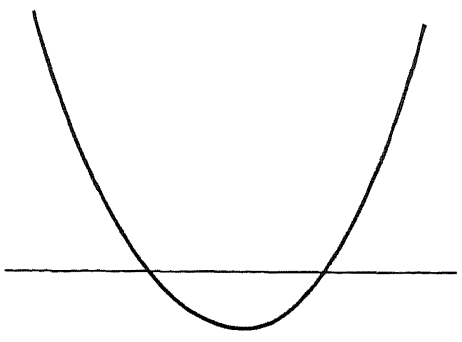

convex function

Fig. 5. A concave and a convex function.

for $x, y \in \mathbb{R}^{d}$ such that $f(x), f(y)<+\infty$ and $0 \leqslant r \leqslant 1$. See Fig. 5 for an illustration.

The concave and convex functions are denoted by Fun $_{\wedge}\left(\mathbb{R}^{d}\right)$ and Fun $\vee\left(\mathbb{R}^{d}\right)$, respectively. Note that the subscript ' $\wedge$ ' characterizes the shape of a concave function. The next two results are easy to prove.

\section{Proposition 3.5.}

(a) If $f$ is concave, then $\operatorname{dom}_{\vee}(f)$ is a convex set. (b) If $f$ is convex, then $\operatorname{dom}_{\wedge}(f)$ is a convex set.

\section{Proposition 3.6.}

(a) $f$ is concave iff $U_{\wedge}(f)$ is a convex set in $\mathbb{R}^{d} \times \mathbb{R}$. (b) $f$ is convex iff $U_{\vee}(f)$ is a convex set in $\mathbb{R}^{d} \times \mathbb{R}$.

Concavity and convexity are dual notions in the sense that $f$ is concave iff $-f$ is convex. There is a huge literature on convex functions; we refer in particular to the monographs of Rockafellar [22] and Van Tiel [30], and the two recent volumes by HiriartUrruty and Lemaréchal [13, 14].

As grey-scale morphology is usually based on the notion of the hypograph (or umbra; this is convex if the underlying function is concave) we choose to consider concave rather than convex functions. From the duality principle [11], it follows that both approaches are equivalent.

The infimum of an arbitrary collection of concave functions is concave. This does not hold for the supremum. Define the concave hull $\beta_{\wedge}(f)$ of an arbitrary function $f$ as the infimum of all concave functions which lie above $f$. This is a concave function, the smallest concave function above $f$. Dually, we define the convex hull $\alpha_{\mathrm{V}}(f)$ as the supremum of all convex functions below $f$. In Fig. 6 we give an illustration of the concave hull.
The next two results are very similar to Propositions 3.3-3.4.

\section{Proposition 3.7.}

(a) The mapping $\beta_{\wedge}$ defines a closing on Fun $\left(\mathbb{R}^{d}\right)$ with invariance domain Fun $_{\wedge}\left(\mathbb{R}^{d}\right)$.

(b) The mapping $\alpha_{\vee}$ defines an opening on $\operatorname{Fun}\left(\mathbb{R}^{d}\right)$ with invariance domain Fun $\checkmark\left(\mathbb{R}^{d}\right)$.

\section{Proposition 3.8.}

(a) The set Fun $\wedge\left(\mathbb{R}^{d}\right)$ is a complete lattice under the pointwise ordering, with the pointwise infimum $\bigwedge_{i \in I} f_{i}$ and with supremum $\beta_{\wedge}\left(\bigvee_{i \in I} f_{i}\right)$.

(b) The set $\operatorname{Fun}_{\vee}\left(\mathbb{R}^{d}\right)$ is a complete lattice under the pointwise ordering, with the pointwise supremum $\bigvee_{i \in I} f_{i}$ and with infimum $\alpha_{\vee}\left(\bigwedge_{i \in I} f_{i}\right)$

The lower closed hull of a convex function is convex [22]. This means that

$\alpha_{\vee} \alpha, \alpha_{\vee}=\alpha_{1} \alpha_{\vee}$

Now Proposition 2.3(a) gives that $\alpha_{f} \alpha_{\vee}$ is an opening with invariance domain $\operatorname{Fun}_{l}\left(\mathbb{R}^{d}\right) \cap \mathrm{Fun}_{\vee}\left(\mathbb{R}^{d}\right)$, the 1.s.c. convex functions. A dual result holds for the upper closed hull of concave functions, i.e.

$\beta_{\wedge} \beta_{\mathrm{u}} \beta_{\wedge}=\beta_{\mathrm{u}} \beta_{\wedge}$

\section{Proposition 3.9.}

(a) The operator $\alpha_{1} \alpha_{V}$ is an opening on Fun $\left(\mathbb{R}^{d}\right)$ with invariance domain the l.s.c. convex functions.

(b) The operator $\beta_{\mathrm{u}} \beta_{\wedge}$ is a closing on $\operatorname{Fun}\left(\mathbb{R}^{d}\right)$ with invariance domain the u.s.c. concave functions. 


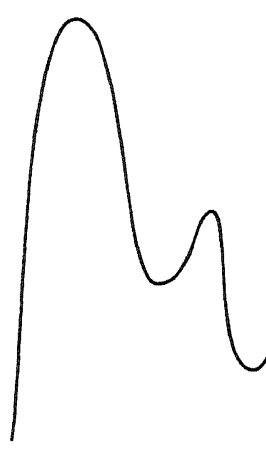

(a)

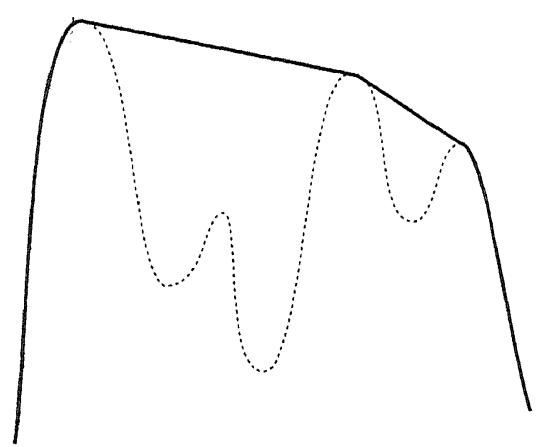

(b)

Fig. 6. (a) A function and (b) its concave hull.

One can find examples which show that $\alpha_{f} \alpha_{\vee} \alpha_{f} \neq$ $\alpha_{\vee} \alpha_{,}$and that $\beta_{\mathrm{u}} \beta_{\wedge} \beta_{\mathrm{u}} \neq \beta_{\wedge} \beta_{\mathrm{u}}$; cf. (2.6).

Remark 3.10. In Fig. 2 we have given an example of a closed set $X$ for which $\operatorname{co}(X)$ is not closed. Similarly, we find that $f$ u.s.c. does not necessarily imply that $\beta_{\wedge}(f)$ is u.s.c. One can show that, for every function $f$, $\overline{\mathrm{co}}\left(U_{\wedge}(f)\right)=U_{\wedge}\left(\beta_{\mathrm{u}} \beta_{\wedge}(f)\right)$.

Refer to [13, Section IV.2.5] for similar results.

In the previous section we have introduced some operations on functions such as supremal and infimal convolution. For these operations, one has to take into account the arithmetical conventions for extended reals as explained in Remark 2.4. This means in particular that ' $\oplus$ ' and ' $\square$ ' are not commutative in all cases.

It is evident that, for every function $y$,

(i) $f \oplus g$ is convex, if $f$ is convex;

(ii) $f \ominus g$ is concave, if $f$ is concave;

(iii) $f \square g$ is concave, if $f$ is concave.

But, more interestingly, one can also prove the following result. Refer to [22, Section 9] and [13, Section. IV.2.3] for some closely related results.

\section{Proposition 3.11.}

(a) If $f, g$ are concave, then $f \oplus g$ is concave.

(b) If $f, g$ are convex, then $f \square g$ is convex.

Proof. We prove (a); then (b) follows from a duality argument. We use the umbra transform discussed in [10] and [11, Section 11.6]. Recall that a set $U \subseteq \mathbb{R}^{d} \times \mathbb{R}$ is called an umbra if $(x, t) \in U \Leftrightarrow(x, s) \in U$ for $s<t$; here $x \in \mathbb{R}^{d}$. The set $U$ is called a pre-umbra if $(x, t) \in U$ implies that $(x, s) \in U$ for $s<t$. For a set $V \subseteq \mathbb{R}^{d} \times \mathbb{R}$ we denote by $U_{\mathrm{s}}(V)$ the smallest umbra which contains $V$. If $V$ is a pre-umbra, then $U_{\mathrm{s}}(V)=\bigcap_{\tau>0} V^{\tau}$. Here $V^{\tau}=\{(x, t+\tau) \mid(x, t) \in V\}$. It is easy to show (see also [11, Section 11.6]) that

$U_{\wedge}(f \oplus g)=U_{\mathrm{s}}\left(U_{\wedge}(f) \oplus U_{\wedge}(g)\right)$

If $f, g$ are concave, then $U_{\wedge}(f), U_{\wedge}(g)$ are convex sets. Therefore (see Section 2.1) their Minkowski sum $U_{\wedge}(f) \oplus U_{\wedge}(g)$ is convex, too. But now $U_{\mathrm{s}}\left(U_{\wedge}(f) \oplus\right.$ $U_{\wedge}(g)$ ), being an intersection of convex sets, is convex. This implies that $U_{\wedge}(f \oplus g)$ is convex, in other words, that $f \oplus g$ is concave.

\subsection{Legendre transform and conjugation}

Consider a convex function $f: \mathbb{R}^{d} \rightarrow \overline{\mathbb{R}}$ which is continuously differentiable and finite. Refer to $[22$, Section 26] for conditions on $f$ which are slightly more general, and under which the derivations below are still valid.

Given a vector $v \in \mathbb{R}^{d}$, we look for a point $x_{0} \in \mathbb{R}^{d}$ such that the hyperplane in $\mathbb{R}^{d} \times \mathbb{R}$ given by $x \mapsto\langle x-$ $\left.x_{0}, v\right\rangle+f\left(x_{0}\right)$ is tangent to the graph of $f$ at the point $x=x_{0}$. This amounts to solving the equation $\nabla f(x)=v$, where $\nabla f$ is the gradient of $f$. If this gradient mapping has an inverse $(\nabla f)^{-1}$, then the solution is given by $x=x(v):=(\nabla f)^{-1}(v)$. It turns out that $x(\cdot)$ is a gradient mapping itself: $x(v)=\nabla F(v)$, where 
$F$ is given by

$$
\begin{aligned}
F(v) & =\langle x(v), v\rangle-f(x(v)) \\
& =\left\langle(\nabla f)^{-1}(v), v\right\rangle-f\left((\nabla f)^{-1}(v)\right) .
\end{aligned}
$$

The function $F$ is called the Legendre transform of $f$. It is well-defined if $f$ is convex and differentiable, and if $\nabla f$ is invertible. For a function $f$ and its Legendre transform $F$, the following inverse relations hold:

$\nabla f(x)=v$ and $\nabla F(v)=x$

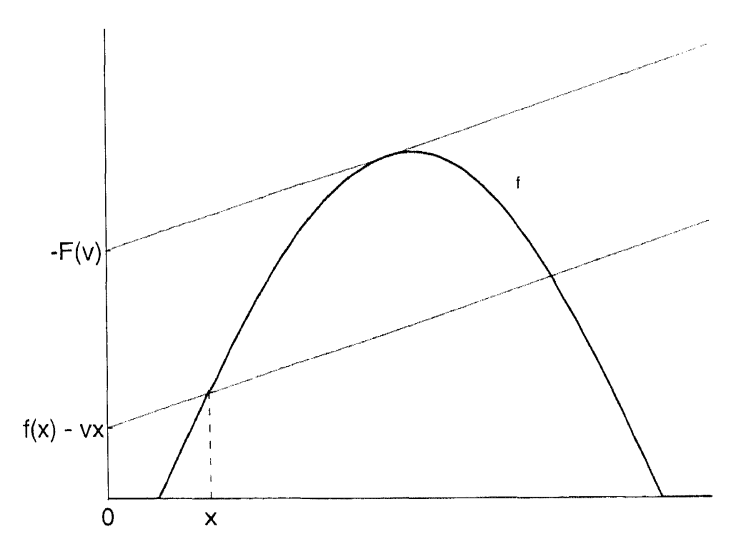

Fig. 7. Concave signal $f$, its tangent with slope $=v$ and a line parallel to the tangent.
Note, in particular, that these relations imply that $f$ is the Legendre transform of $F$. If $x, v$ are related by (3.4), then

$$
f(x)+F(v)=\langle x, l\rangle .
$$

As shown in Fig. 7 for a one-dimensional differentiable signal $f$, the quantity $l x-f(x)$ is the negative of the intercept of a line that passes from the point $(x, f(x))$ on the graph of $f$ and has slope $t$. This intercept becomes maximum (and equal to $-F(v)$ ) when the line with slope $v$ becomes tangent to the graph of $f$. In Fig. 8 we depict an example.

If $f$ does not have an invertible gradient, its Legendre transform cannot be defined as above. To treat such and other more general cases of non-differentiable functions, we now define $F$ as

$$
F(v)=-\bigwedge_{x \in \mathbb{R}^{d}} f(x)-\langle x, v\rangle=\bigvee_{x \in \mathbb{R}^{d}}\langle x, v\rangle-f(x) .
$$

The conjugate $f^{\star}$ of a function $f$ is defined by

$$
f^{\star}(v)=\bigvee_{x \in \mathbb{R}^{\prime}}\langle x, v\rangle-f(x)
$$

We write $A(f)=f^{\star}$. The operator $A$ is known under different names, e.g. 'Fenchel conjugate', 'YoungFenchel conjugate', or 'Legendre-Fenchel transform'; see e.g. $[22,13,14]$.
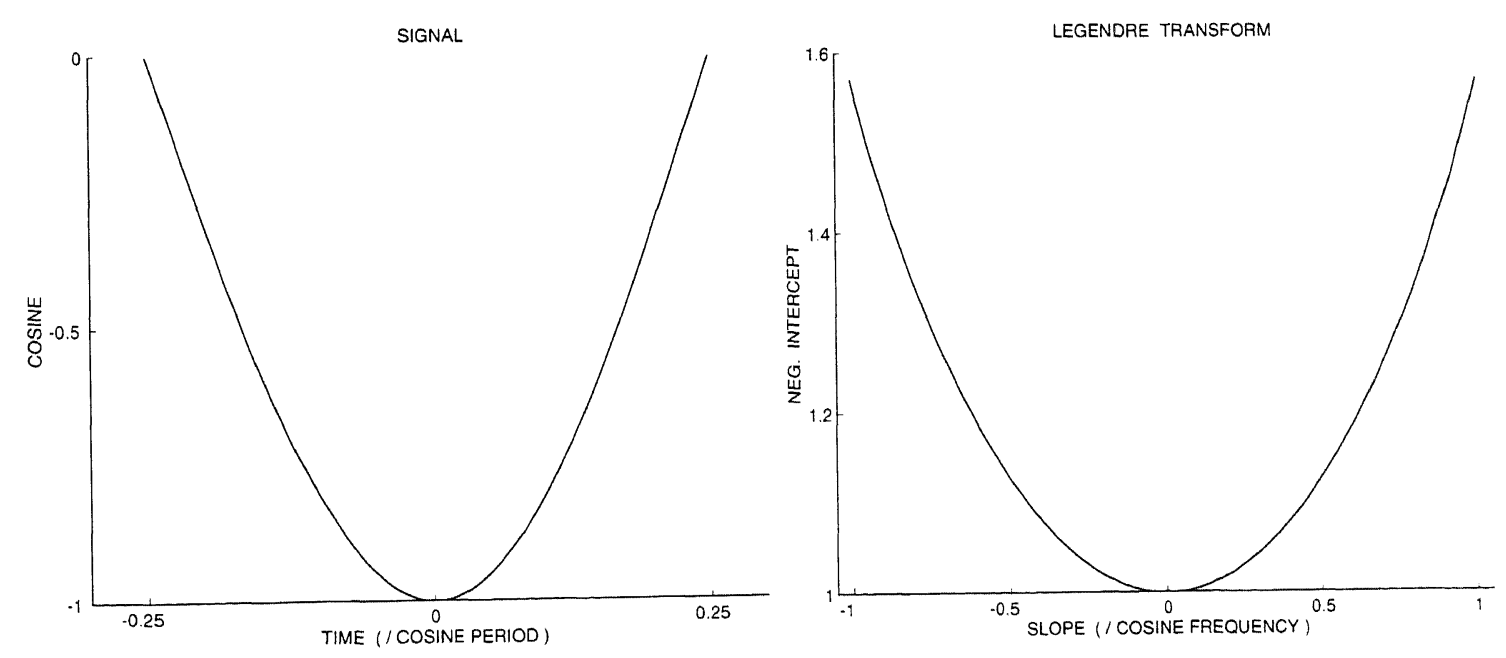

Fig. 8. (a) Signal $f(x)=-\cos \left(\omega_{0} x\right),|x| \leqslant \pi /\left(2 \omega_{0}\right)$. (b) Its Legendre transform $F(v)=\sqrt{1-\left(c / \omega_{0}\right)^{2}}+\left(v / \omega_{0}\right) \arcsin \left(c / \omega_{0}\right)$. 
The definition in (3.6) is not restricted to convex functions but applies to all functions $f: \mathbb{R}^{d} \rightarrow \overline{\mathbb{R}}$. Note that $f^{\star}$ is identically $+\infty$ if $f^{\prime}(x)=-\infty$ for some $x \in \mathbb{R}^{d}$. From (3.6) it follows readily that

$f^{\star}(v) \geqslant\langle x, v\rangle-f(x)$,

for every $x \in \mathbb{R}^{d}$ and $v \in \mathbb{R}^{d}$. This inequality is known as Fenchel's inequality, and is usually written as $f(x)+f^{\star}(v) \geqslant\langle x, v\rangle$. However, this latter inequality may differ from (3.7) if $f(x)$ or $f^{\star}(v)$ equal $\pm \infty$.

The next result can be found in [22] for the case where $f$ is a convex function.

Proposition 3.12. For every $f \in \mathrm{Fun}\left(\mathbb{R}^{d}\right)$, the conjugate $f^{\star}$ is l.s.c. and convex.

Proof. Formula (3.6) shows that $f^{\star}$ is the supremum of affine functions $x \mapsto\langle x, v\rangle-f(x)$. From Proposition 3.4(b) we find that $f^{\star}$ is 1.s.c., and from Proposition $3.8\left(\right.$ b) we conclude that $f^{\star}$ is convex.

At this point we might give a list of properties of the conjugation. However, this operation is closely related to the slope transforms discussed later. As we are primarily concerned with the slope transforms, we rather discuss properties of the latter. We mention only the property that conjugation transforms an infimal convolution into an addition:

$(f \square g)^{\star}=f^{\star}+g^{\star}$,

for $f, g \in \operatorname{Fun}\left(\mathbb{R}^{d}\right)$.

\subsection{Upper slope transform}

Recall the following definition of the upper slope transform from Section 2.4:

$f^{\vee}(v)=\mathscr{S}_{\vee}(f)(v)=\bigvee_{x \in \mathbb{R}^{\prime}} f(x)-\langle x, v\rangle$,

for $f \in$ Fun $\left(\mathbb{R}^{d}\right)$. See Fig. 9 for examples of slope transforms of differentiable and non-differentiable signals.

There exists a simple relationship between this transform and the Young-Fenchel conjugate, namely,

$f^{\vee}(v)=(-f)^{\star}(-v)$.

This relation, in combination with Proposition 3.12 yields the following result.

Proposition 3.13. For every $f \in \operatorname{Fun}\left(\mathbb{R}^{d}\right)$, its upper slope transform $f^{\vee}$ is l.s.c. and convex.

We list a number of properties of the upper slope transform; see also [18]. Define for $f \in \operatorname{Fun}\left(\mathbb{R}^{d}\right)$ and
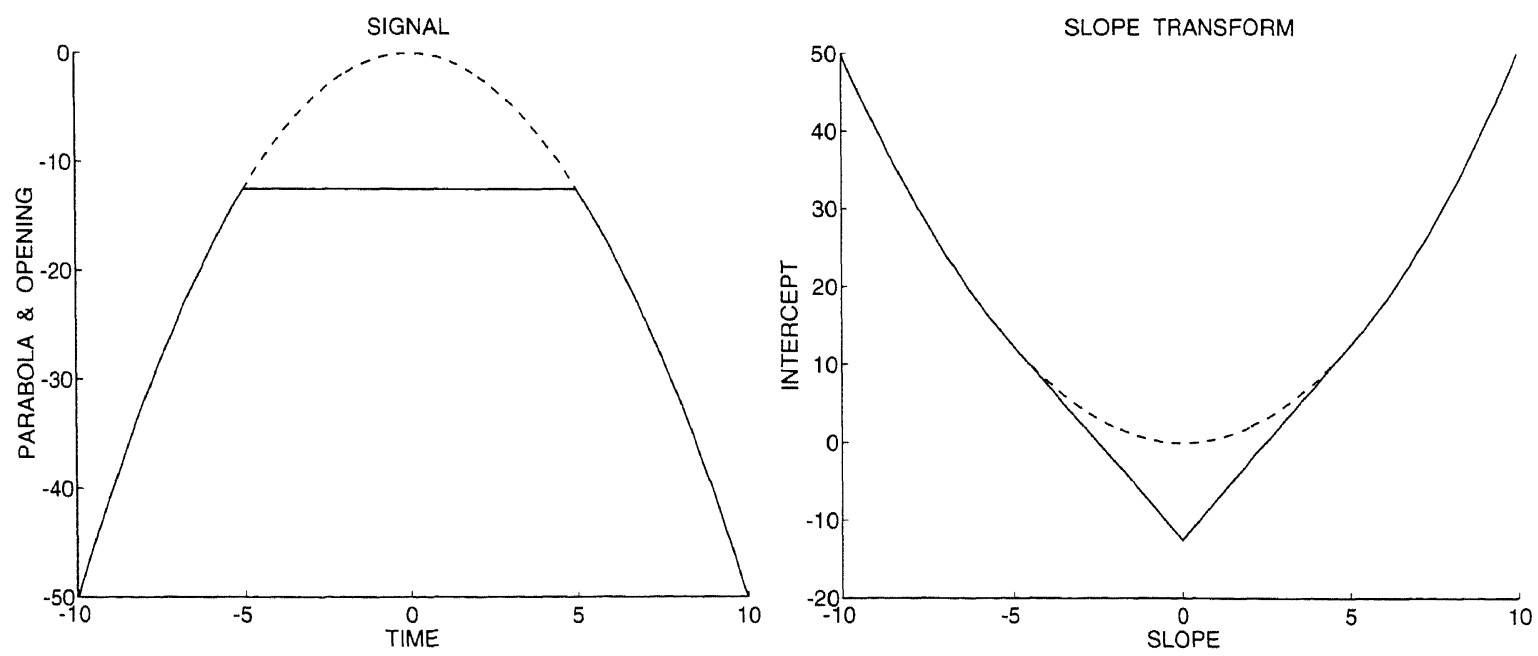

Fig. 9. (a) Original parabola signal $f(x)=-x^{2} / 2$ (in dashed line) and its morphological opening (in solid line) by a flat structuring element $[-5,5]$. (b) Upper slope transform of the parabola (in dashed line) and of its opening (in solid line). 
$w \in \mathbb{R}^{d}$,

$f_{[w]}(x)=f(x)+\langle x, w\rangle$.

The notation $f(r \cdot)$, where $r \in \mathbb{R}$, stands for the function $x \mapsto f(r x)$.

Proposition 3.14. (Properties of $\mathscr{S}_{V}$ ). For $f, g \in$ Fun $\left(\mathbb{R}^{d}\right), y \in \mathbb{R}^{d}, w \in \mathbb{R}^{d}, r>0$, and $c \in \overline{\mathbb{R}}$ :

(a) $\left(f_{y}\right)^{\vee}=\left(f^{\vee}\right)_{[-y]}$,

(b) $\left(f_{[w]}\right)^{\vee}=\left(f^{\vee}\right)_{w}$,

(c) $(f+c)^{\vee}=f^{\vee}+c$

(d) $(r f)^{\vee}=r f^{\vee}(\cdot / r)$

(e) $f(r \cdot)^{\vee}=f^{\vee}(\cdot / r)$,

(f) $f(-\cdot)^{\vee}=f^{\vee}(-\cdot)$,

(g) $(f \oplus g)^{\vee}=f^{\vee}+g^{\vee}$.

If $f^{\vee}(v)=b$, then the function $f$ is majorized by the affine function $x \mapsto\langle x, v\rangle+b$. Therefore, if we compute the infimum of all affine functions $x \mapsto\langle x, v\rangle+f^{\vee}(v)$, we obtain a function which majorizes the original function $f$. This motivates us to define

$\mathscr{S}_{\vee}^{\leftarrow}(g)(x)=\bigwedge_{v \in \mathbb{R}^{d}} g(v)+\langle x, v\rangle$,

for a function $g: \mathbb{R}^{d} \rightarrow \overline{\mathbb{R}}$.

The upper slope transform maps the affine function $x \mapsto\left\langle x, v_{0}\right\rangle+b$ onto an upper impulse which equals $b$ for $v=v_{0}$ and $+\infty$ elsewhere. If we apply $\mathscr{S}_{V}^{\leftarrow}$ to this upper impulse, we retrieve the original input function $x \mapsto\left\langle x, v_{0}\right\rangle+b$.

We call $\mathscr{S}_{V}^{\leftarrow}$ the adjoint upper slope transform. This nomenclature is justified by our next result.

Proposition 3.15. $\left(\mathscr{S}_{\vee}^{\leftarrow}, \mathscr{S}_{\vee}\right)$ is an adjunction on Fun $\left(\mathbb{R}^{d}\right)$.

Proof. We must show that

$\mathscr{S}_{\vee}(f) \leqslant g \Leftrightarrow f \leqslant \mathscr{S}_{\vee}^{\leftarrow}(g)$.

We prove ' $\Rightarrow$ '; the other implication is proved similarly. Assume that $\mathscr{S}_{\vee}(f) \leqslant g$; this means that

$f(x)-\langle x, v\rangle \leqslant g(v), \quad x \in \mathbb{R}^{d}, v \in \mathbb{R}^{d}$.

Therefore, $f(x) \leqslant g(v)+\langle x, v\rangle$ for $x \in \mathbb{R}^{d}, v \in \mathbb{R}^{d}$. This yields that $f(x) \leqslant \bigwedge_{v \in \mathbb{B}^{d}} g(v)+\langle x, v\rangle$ for $x \in \mathbb{R}^{d}$, i.e., $f \leqslant \mathscr{S}_{V}^{\leftarrow}(g)$.
Analogous to Proposition 3.13 we can prove that the function $\mathscr{S}_{\vee}^{\leftarrow}(g)$ is u.s.c. and concave for an arbitrary function $g$. In fact, we can prove a much stronger result.

\section{Proposition 3.16.}

(a) $\operatorname{Ran}\left(\mathscr{S}_{V}\right)$ consists of the l.s.c. convex functions.

(b) $\operatorname{Ran}\left(\mathscr{S}_{\vee}^{\leftarrow}\right)$ consists of the u.s.c. concave functions.

Proof. We prove (b); the proof of (a) follows by similar arguments. Assume that $f$ is u.s.c. and concave; we show that $f \in \operatorname{Ran}\left(\mathscr{S}_{\vee}^{-}\right)$. Define $g=\mathscr{S}_{\vee}(f)$; we show that $\mathscr{S}_{V}^{\leftarrow}(g)=f$. Put $f^{\prime}=\mathscr{S}_{V}^{\leftarrow}(g)$. Since $\mathscr{S}_{V}^{\leftarrow} \mathscr{R}_{V}$ is a closing we get that $f^{\prime}=\mathscr{S}_{\vee} \mathscr{S}_{\vee}(f) \geqslant f$. Therefore, it remains to be shown that $f \geqslant f^{\prime}$. Since $f$ is u.s.c. and concave, it follows that $f$ is the infimum of all affine functions $x \mapsto\langle x, v\rangle+b$ majorizing $f$; $\mathrm{cf}$. [13, Proposition IV.1.2.8]. If $\ell$ is such an affine function, then $\ell=\mathscr{S}_{v}-\mathscr{S}_{v}(\ell) \geqslant \mathscr{S}_{v} \leftarrow \mathscr{S}_{V}(f)=f^{\prime}$. But this implies immediately that $f \geqslant f^{\prime}$.

Combining the latter two propositions we arrive at the following result.

\section{Corollary 3.17.}

(a) $\mathscr{S}_{\vee}^{\leftarrow} \mathscr{S}_{V}$ is a closing on $\operatorname{Fun}\left(\mathbb{R}^{d}\right)$ with invariance domain the u.s.c. concave functions, i.e.,

$\mathscr{S}_{V}^{\leftarrow} \mathscr{S}_{V}=\beta_{\mathrm{u}} \beta_{\wedge}$.

(b) $\mathscr{S}_{\vee} \mathscr{S}_{\vee}^{\leftarrow}$ is an opening on Fun $\left(\mathbb{R}^{d}\right)$ with invariance domain the l.s.c. convex functions, i.e.,

$$
\mathscr{S}_{V} \mathscr{S}_{V}^{\leftarrow}=\alpha_{\ell} \alpha_{V} \text {. }
$$

If we apply Proposition 2.1(b) to the adjunction $\left(\mathscr{S}_{V}^{\leftarrow}, \mathscr{S}_{V}\right)$, we find that

$\mathscr{S}_{\vee}\left(\bigwedge_{j \in J} f_{j}\right)=\alpha_{\ell} \alpha_{\vee}\left(\bigwedge_{j \in J} \mathscr{S}_{\vee}\left(f_{j}\right)\right)$,

if $f_{j}$ is u.s.c. and concave for every $j \in J$.

We now list a number of properties of $\mathscr{S}_{V}^{\leftarrow}$; see also [18].

Proposition 3.18. (Properties of $\mathscr{S}_{\vee}^{\leftarrow}$ ). For $f, g \in$ Fun $\left(\mathbb{R}^{d}\right), y \in \mathbb{R}^{d}, w \in \mathbb{R}^{d}, r>0$, and $c \in \overline{\mathbb{R}}$ :

(a) $\mathscr{S}_{\vee}^{\leftarrow}\left(f_{w}\right)=\left(\mathscr{S}_{\vee}^{\leftarrow}(f)\right)_{[w]}$, 

(b) $\mathscr{S}_{\vee}\left(f_{[y]}\right)=\left(\mathscr{S}_{\vee}^{\leftarrow}(f)\right)_{-y}$,
(c) $\mathscr{P}_{\checkmark}^{\leftarrow}(f+c)=\mathscr{S}_{V}^{\leftarrow}(f)+c$,
(d) $\mathscr{S}_{\checkmark}^{\leftarrow}(r f)=r \mathscr{S}_{\checkmark}(f)(\cdot / r)$,
(e) $\mathscr{S}_{\vee}^{\leftarrow}(f(r \cdot))=\mathscr{S}_{\vee}^{-}(f)(\cdot / r)$
(f) $\mathscr{S}_{\vee}^{\leftarrow}(f(-\cdot))=\mathscr{S}_{\vee}^{\leftarrow}(f)(-\cdot)$
(g) $\mathscr{P}_{\vee}(f \square g)=\mathscr{S}_{\checkmark}(f)+\mathscr{S}_{\vee}(g)$.

Further, it is easy to verify that

$\mathscr{S}_{\vee}^{\leftarrow}(-f)=-\mathscr{S}_{\vee}(f) ;$

in other words, $\mathscr{S}_{v}^{-}$is the negative operator of $\mathscr{S}_{V}$. However, we point out that (3.12) is, in a sense, meaningless. For, the upper slope transform acts on functions of the spatial variable $x$, whereas the adjoint upper slope transform acts on functions of the slope variable $v$.

\subsection{Lower slope transform}

If we replace the supremum in (3.9) by an infimum we get the lower slope transform. It goes without saying that all results for the upper slope transform stated in the previous section have a counterpart for the lower slope transform. For the sake of completeness we will state them briefly.

Let $f \in \operatorname{Fun}\left(\mathbb{R}^{d}\right)$, the lower slope transform of $f$ is

$$
f^{\wedge}(v)=\mathscr{S}_{\wedge}(f)(v)=\bigwedge_{x \in \mathbb{R}^{d}} f(x)-\langle x, v\rangle .
$$

There exists the following relationship with the upper slope transform and the Young-Fenchel conjugate:

$f^{\wedge}(v)=-f^{\star}(v)=-(-f)^{\vee}(-v)$.

Proposition 3.19. For every $f \in \operatorname{Fun}\left(\mathbb{R}^{d}\right)$, its lower slope transform $f^{\wedge}$ is u.s.c. and concave.

We list a number of properties of the lower slope transform; cf. [18].

Proposition 3.20. (Properties of $\mathscr{S}_{\wedge}$ ). For $f, g \in$ Fun $\left(\mathbb{R}^{d}\right), y \in \mathbb{R}^{d}, w \in \mathbb{R}^{d}, r>0$ and $c \in \overline{\mathbb{R}}$ :
(a) $\left(f_{y}\right)^{\wedge}=\left(f^{\wedge}\right)_{[-y]}$,
(b) $\left(f_{[w]}\right)^{\wedge}=\left(f^{\wedge}\right)_{w}$,
(c) $(f+c)^{\wedge}=f^{\wedge}+c$,
(d) $(r f)^{\wedge}=r f^{\wedge}(\cdot / r)$,
(e) $f(r \cdot)^{\wedge}=f^{\wedge}(\cdot / r)$,

(f) $f(-\cdot)^{\wedge}=f^{\wedge}(-\cdot)$,

(g) $(f \square g)^{\wedge}=f^{\wedge}+g^{\wedge}$.

Analogous to (3.11) we define

$\mathscr{S}_{\wedge}^{\leftarrow}(g)(x)=\bigvee_{v \in \mathbb{R}^{d}} g(v)+\langle x, v\rangle$,

which we call the adjoint lower slope transform. We now state without proof the analogues of Propositions 3.15 and 3.16 and Corollary 3.17.

Proposition 3.21. $\left(\mathscr{S}_{\wedge}, \mathscr{S}_{\wedge}^{\leftarrow}\right)$ is an adjunction on $\operatorname{Fun}\left(\mathbb{R}^{d}\right)$.

\section{Proposition 3.22.}

(a) $\operatorname{Ran}\left(\mathscr{S}_{\wedge}\right)$ consists of the $u . s . c$. concave functions.

(b) $\operatorname{Ran}\left(\mathscr{S}_{\wedge}^{\leftarrow}\right)$ consists of the l.s.c. convex functions.

\section{Corollary 3.23.}

(a) $\mathscr{S}_{\wedge}^{\leftarrow} \mathscr{S}_{\wedge}$ is an opening on Fun $\left(\mathbb{R}^{d}\right)$ with invariance domain the l.s.c. convex functions, i.e.,

$\mathscr{S}_{\wedge}^{\leftarrow} \mathscr{S}_{\wedge}=\alpha_{\ell} \alpha_{\vee}$.

(b) $\mathscr{S}_{\wedge} \mathscr{S}_{\wedge}^{\leftarrow}$ is a closing on Fun $\left(\mathbb{R}^{d}\right)$ with invariance domain the u.s.c. concave functions, i.e.,

$$
\mathscr{S}_{\wedge} \mathscr{S}_{\wedge}^{\leftarrow}=\beta_{\mathrm{u}} \beta_{\wedge} .
$$

We state some properties of $\mathscr{S}_{\wedge}^{\leftarrow}$; cf. [18].

Proposition 3.24. (Properties of $\mathscr{S}_{\wedge}^{\leftarrow}$ ). For $f, g \in$ Fun $\left(\mathbb{R}^{d}\right), y \in \mathbb{R}^{d}, w \in \mathbb{R}^{d}, r>0$, and $c \in \overline{\mathbb{R}}$ :
(a) $\mathscr{S}_{\wedge}^{\leftarrow}\left(f_{w}\right)=\left(\mathscr{S}_{\wedge}^{\leftarrow}(f)\right)_{[w]}$,
(b) $\mathscr{S}_{\wedge}\left(f_{[y]}\right)=\left(\mathscr{S}_{\wedge}^{\leftarrow}(f)\right)_{-y}$,
(c) $\mathscr{S}_{\wedge}^{\leftarrow}(f+c)=\mathscr{S}_{\wedge}^{\leftarrow}(f)+c$
(d) $\mathscr{S}_{\wedge}^{\leftarrow}(r f)=r \mathscr{S}_{\wedge}^{\leftarrow}(f)(\cdot / r)$,
(e) $\mathscr{S}_{\wedge}^{\leftarrow}(f(r \cdot))=\mathscr{S}_{\wedge}^{\leftarrow}(f)(\cdot / r)$,
(f) $\mathscr{S}_{\wedge}^{\leftarrow}(f(-\cdot))=\mathscr{S}_{\wedge}^{\leftarrow}(f)(-\cdot)$
(g) $\mathscr{S}_{\wedge}^{\leftarrow}(f \oplus g)=\mathscr{S}_{\wedge}^{\leftarrow}(f)+\mathscr{S}_{\wedge}^{\leftarrow}(g)$.

\section{Slope transform for sets}

\subsection{Preparations}

In Section 2.1 we have summarized some basic facts about convex sets. In this preparatory section we present some additional notations. 
We can embed the convex sets into the lattice of concave (respectively convex) functions. Thereto we need the following definitions. The upper and lower indicator function corresponding to a set $X$ are defined as

$l \vee(X)(x)= \begin{cases}0, & x \in X, \\ +\infty, & x \notin X,\end{cases}$

and

$l_{\wedge}(X)(x)= \begin{cases}0, & x \in X, \\ -\infty, & x \notin X,\end{cases}$

respectively. It is evident that

$X$ closed $\Leftrightarrow l_{\vee}(X)$ l.s.c. $\Leftrightarrow l_{\wedge}(X)$ u.s.c.

$X$ convex $\Leftrightarrow l_{\vee}(X)$ convex $\Leftrightarrow l_{\wedge}(X)$ concave.

We introduce some further notation; see [24]. We denote, for $a \in \mathbb{R}^{d}$ and $r \in \mathbb{R}$, by $\mathbb{H}(a, r)$ the hyperplane

$\mathrm{H}(a, r)=\left\{x \in \mathbb{R}^{d} \mid\langle a, x\rangle=r\right\}$.

Note that $\mathbb{H}(a, r)=\emptyset$ if $r= \pm \infty$. Furthermore, $\mathrm{H}^{-}(a, r)$ and $\mathbb{H}^{+}(a, r)$ are the closed half spaces

$\mathbb{H}^{-}(a, r)=\left\{x \in \mathbb{R}^{d} \mid\langle a, x\rangle \leqslant r\right\}$,

$\mathbb{H}^{+}(a, r)=\left\{x \in \mathbb{R}^{d} \mid\langle a, x\rangle \geqslant r\right\}$.

If $r=-\infty$ then $\mathbb{H}^{-}(a, r)=\emptyset$ and $\mathbb{H}^{+}(a, r)=\mathbb{R}^{d}$; dually, if $r=+\infty$ then $\mathbb{H}^{-}(a, r)=\mathbb{R}^{d}$ and $\mathbb{H}^{+}(a, r)=\emptyset$. We say that the hyperplane $H(a, r)$ supports the set $X \subseteq \mathbb{R}^{d}$ at $h$ if $h \in X \cap \mathbb{H}(a, r)$ and $X \subseteq \mathbb{H}^{-}(a, r)$ or $X \subseteq \mathbb{H}^{+}(a, r)$.

\subsection{Sublinear functions}

We start with a definition.

Definition 4.1. A function $f: \mathbb{R}^{d} \rightarrow \overline{\mathbb{R}}$ is said to be positively homogeneous if $f(r x)=r f(x)$ for $r>0$ and $x \in \mathbb{R}^{d}$. It is sublinear if it is both convex and positively homogeneous.

For a comprehensive discussion on sublinear functions the reader may refer to [13, Chapter V]. It is easy to see that any sublinear function satisfies $f(0)=0,-\infty$ or $+\infty$. Note that $f \equiv-\infty$ if $f(0)=-\infty$. Furthermore, the epigraph $U_{\vee}(f)$ is a convex cone. Every sublinear function satisfies the inequality

$f(x+y) \leqslant f(x)+f(y)$

a function with this property is called subadditive. We give some examples.

\section{Examples 4.2.}

(a) If $K \subseteq \mathbb{R}^{d}$ is a convex cone, then the upper indicator function $l \vee(K)$ is sublinear

(b) A function $\|\cdot\|: \mathbb{R}^{d} \rightarrow \overline{\mathbb{R}}_{+}=[0,+\infty]$ is called a norm if

(i) $\|x\|=0$ iff $x=0$;

(ii) $\|r x\|=|r| \cdot\|x\|, r \in \mathbb{R}, x \in \mathbb{R}^{d}$;

(iii) $\|x+y\| \leqslant\|x\|+\|y\|, x, y \in \mathbb{R}^{d}$.

Note that $\|x\|$ is allowed to be $+\infty$. Every norm is a (nonnegative) sublinear function.

(c) Let $X \subseteq \mathbb{R}^{d}$ be a convex set containing the origin. The function $\gamma(X)$ defined by

$\gamma(X)(x)=\inf \{r>0 \mid x \in r X\}$

is called the gauge (function) of $X$. It is a sublinear function.

We prove the following lemma.

Lemma 4.3. If $f$ is positively homogeneous then its convex hull $\alpha_{\vee}(f)$ is positively homogeneous as well.

Proof. Define, for a function $f$ and a real number $r>0, f_{r}(x)=r f(x / r)$. Thus, $f$ is positively homogeneous iff $f_{r}=f$ for every $r>0$. The convex hull of a function $f$ is given by

$\alpha_{\vee}(f)=\bigvee_{g \in f_{i}} g$,

where $\mathscr{C}$ consists of all convex functions $g \leqslant f$. It follows immediately that $g \in \mathscr{C} \Rightarrow g_{r} \in \mathscr{C}$ for every $r>0$. But this implies that $x_{\vee}(f)$ is positively homogeneous, too.

We denote the family of all sublinear functions by $\operatorname{Fun}_{\text {sl }}\left(\mathbb{R}^{d}\right)$. It is easy to verify that the pointwise supremum of an arbitrary collection $f_{i}, i \in I$, of sublinear functions is sublinear. Thus there exists an opening $\alpha_{\mathrm{sl}}$ on $\operatorname{Fun}\left(\mathbb{R}^{d}\right)$ with invariance domain $\mathrm{Fun}_{\mathrm{sl}}\left(\mathbb{R}^{d}\right)$.

The pointwise infimum of a collection of sublinear functions is positively homogeneous but not 
subadditive in general. Let $f \in \mathrm{Fun}_{\mathrm{sl}}\left(\mathbb{R}^{d}\right)$ such that $f \leqslant f_{i}$ for $i \in I$. Now $\alpha_{\vee}(f)=f$ since $f$ is convex, and we find that $f \leqslant \alpha_{\vee}\left(\bigwedge_{i \in I} f_{i}\right)$. Lemma 4.3 says that $\alpha_{\vee}\left(\bigwedge_{i \in I} f_{i}\right)$ is positively homogeneous, and hence it is sublinear. We conclude that $\alpha_{\vee}\left(\bigwedge_{i \in I} f_{i}\right)$ is the infimum in the complete lattice $\mathrm{Fun}_{\mathrm{sl}}\left(\mathbb{R}^{d}\right)$.

Proposition 4.4. The set $\mathrm{Fun}_{\mathrm{sl}}\left(\mathbb{R}^{d}\right)$ with the pointwise partial ordering is a complete lattice with the usual pointwise supremum and with infimum $\alpha_{\vee}\left(\bigwedge_{i \in I} f_{i}\right)$.

Remark 4.5. If $f, g$ are sublinear and not identically $\pm \infty$, then

$\alpha_{\vee}(f \wedge g)=f \square g$.

To prove $\leqslant$, observe that $(f \square g)(x) \leqslant f(x)+g(0)$ $=f(x)$. Hence $f \square g \leqslant f$ and similarly $f \square g \leqslant g$. This yields that $f \square g \leqslant f \wedge g$. Since $f \square g$ is convex, we get $f \square g \leqslant \alpha_{\vee}(f \wedge g)$.

To show that $f \square g \geqslant h:=\alpha_{\vee}(f \wedge g)$, observe that $h$ is the infimum of $f$ and $g$ in Fun $_{\text {sl }}\left(\mathbb{R}^{d}\right)$, hence $h \leqslant f$ and $h \leqslant g$. This implies that $h \square h \leqslant f \square g$. As $h$ is subadditive, we find that $h(x-y)+h(y) \geqslant h(x)$, and therefore $h \square h \geqslant h$. This proves relation (4.2).

Refer to [13, p. 206] for a different proof.

\subsection{Slope transform for sets: the support function}

For a set $X \subseteq \mathbb{R}^{d}$ its support function $\sigma(X)$ is defined by

$\sigma(X)(v)=\bigvee_{x \in X}\langle x, v\rangle, v \in \mathbb{R}^{d}$.

Note that $\sigma(X) \equiv-\infty$ if $X=\emptyset$. Refer to Fig. 10 for an illustration.

From the observation that the support function is the pointwise supremum of the affine functions $v \mapsto\langle x, v\rangle$, $x \in X$, and Propositions 3.4(b) and 4.4, the following result is clear.

Proposition 4.6. The support function $\sigma(X)$ of a set $X \subseteq \mathbb{R}^{d}$ is l.s.c. and sublinear.

We call the operator $\sigma: \mathscr{P}\left(\mathbb{R}^{d}\right) \rightarrow$ Fun $\left(\mathbb{R}^{d}\right)$, which maps a set $X$ to the corresponding support function, the slope transform for sets.

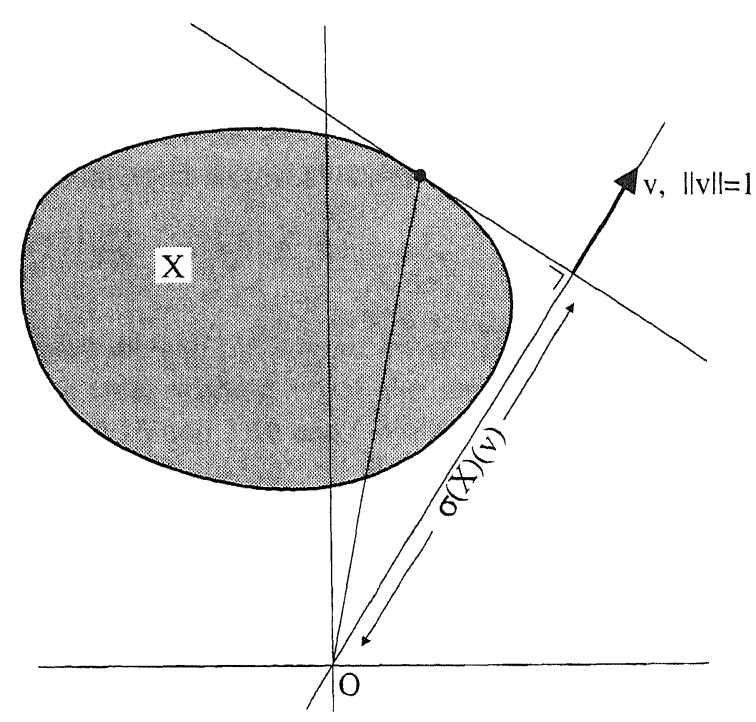

Fig. 10. Support function.

There is a simple correspondence between the slope transform for functions and that for sets, namely,

$$
\begin{aligned}
\mathscr{S}_{V}\left(l_{\wedge}(X)\right)(v) & =\bigvee_{x \in \mathbb{R}^{d}} l_{\wedge}(X)(x)-\langle x, v\rangle \\
& =\bigvee_{x \in X}-\langle x, v\rangle,
\end{aligned}
$$

whence we derive

$\mathscr{S}_{\vee}\left(l_{\wedge}(X)\right)(v)=\sigma(X)(-v)=\sigma(-X)(v)$.

We also have

$\mathscr{S}_{\wedge}(I \vee(X))(v)=\sigma(X)(-v)=\sigma(-X)(v)$

and

$\left(l_{\vee}(X)\right)^{\star}=\sigma(X)$.

Remark 4.7. Strictly speaking, we should refer to $\sigma$ as the 'upper' slope transform. The lower slope transform should then be defined as follows:

$\sigma_{\wedge}(X)(v)=\bigwedge_{x \in X}\langle x, v\rangle$

Such a definition would only make sense if we would introduce the concept of 'concave sets', i.e., complements of convex sets. In order to keep new notation and terminology limited, we will not do so. 
If $\sigma(X)(v)=b$, then $\langle x, v\rangle \leqslant b$ for $x \in X$, that is, $X \subseteq \mathbb{Q}^{-}(v, b)$. We define the operator $\sigma^{-}:$Fun $\left(\mathbb{R}^{d}\right)$ $\rightarrow \mathscr{P}\left(\mathbb{R}^{d}\right)$ by

$$
\sigma^{-}(f)=\bigcap_{v \in \mathbb{R}^{d}} \mathbb{M}^{-}(v, f(v)) .
$$

It is obvious that $\sigma^{\leftarrow}(f)$ is a closed convex set for every function $f$.

Lemma 4.8. Let $X \subseteq \mathbb{R}^{d}, v \in \mathbb{R}^{d}$, and $b \in \overline{\mathbb{R}}$, then

$X \subseteq \mathbb{H}^{-}(v, b)$ iff $\sigma(X)(v) \leqslant b$.

Proof. 'only if': assume $X \subseteq \mathbb{H}^{-}(v, b)$. Then $\sigma(X)(v)=\sup \{\langle x, v\rangle \mid x \in X\} \leqslant b$.

'if': assume $\sigma(X)(v) \leqslant b$. If $x \in X$, then $\langle x, v\rangle \leqslant b$, hence $x \in \mathbb{H}^{-}(v, b)$.

Proposition 4.9. The pair $\left(\sigma^{\leftarrow}, \sigma\right)$ constitutes an adjunction between Fun $\left(\mathbb{R}^{d}\right)$ and $\mathscr{P}\left(\mathbb{R}^{d}\right)$.

Proof. We must show that

$\sigma(X) \leqslant f \Leftrightarrow X \subseteq \sigma^{-}(f)$.

First we prove ' $\Rightarrow$ '. Assume that $\sigma(X) \leqslant f$ and that $x \in X$. We must show that $x \in \mathbb{H}^{-}(v, f(v))$ for $v \in \mathbb{R}^{d}$. This follows from

$\langle x, v\rangle \leqslant \sigma(X)(v) \leqslant f(v)$.

' $\Leftarrow$ ': Assume that $X \subseteq \sigma^{-}(f)=\bigcap_{v \in \mathbb{R}^{d}} \mathbb{H}(v, f(v))$. Thus $X \subseteq \mathbb{H}(v, f(v))$ for $v \in \mathbb{R}^{d}$. From the previous lemma we conclude that $\sigma(X)(v) \leqslant f(v)$, i.e., $\sigma(X) \leqslant f$.

\section{Proposition 4.10.}

(a) $\operatorname{Ran}\left(\sigma^{\leftarrow}\right)$ consists of the closed convex sets in $\mathbb{R}^{d}$.

(b) $\operatorname{Ran}(\sigma)$ consists of the l.s.c. sublinear functions on $\mathbb{R}^{d}$.

Proof. (a) It is evident that every set in $\operatorname{Ran}\left(\sigma^{-}\right)$is closed and convex. On the other hand, if $X$ is closed and convex, then $X$ can be represented as the intersection of all closed halfspaces which contain it.

(b) We have seen that every support function is l.s.c. and sublinear. Assume, on the other hand, that $f$ is 1.s.c. and sublinear. Let $X=\sigma^{-}(f)$, we show that $\sigma(X)=f$. Note first that $\sigma(X)=\sigma \sigma^{-}(f) \leqslant f$, since $\sigma \sigma^{-}$is an opening. Thus it remains to be shown that $\sigma(X) \geqslant f$. The following proof is taken from Schneider [24, 2nd proof of Theorem 1.7.1]. Since $f$ is sublinear and l.s.c. its epigraph $U_{v}(f)$ is a closed convex cone in $\mathbb{R}^{d} \times \mathbb{R}$. Let $v \neq 0$, then $(v, f(v))$ lies on the boundary of $U_{\vee}(f)$. There exists a support plane $\mathbb{H}((y, a), r)$, where $y \in \mathbb{R}^{d-1}$ and $a \in \mathbb{R}$ to $U_{\vee}(f)$ through $(v, f(v))$ such that $U_{\vee}(f) \subseteq \mathbb{t}^{-}((v, a), r)$. This yields that for $t \in \mathbb{R}, w \in \mathbb{R}^{d}$ :

$t \geqslant f(w) \Rightarrow\langle y, w\rangle+a t \leqslant r$.

Since $U_{\mathrm{V}}(f)$ is a cone, the support plane must contain $(0,0)$, hence $r=0$. Suppose that $a \geqslant 0$, then $\langle y, w\rangle \leqslant 0$ for all $w$; this is impossible, hence $a<0$. Without loss of generality, we can assume that $a=-1$. Thus,

$t \geqslant f(w) \Rightarrow\langle y, w\rangle \leqslant t$.

Then $\langle y, w\rangle \leqslant f(w)$ for all $w$. Thus $y \in X$, that is $X \neq \emptyset$. Furthermore, $\langle y, v\rangle=f(v)$ (for $(v, f(v)) \in$ $H((y,-1), 0))$. Then

$\sigma(X)(v)=\sup \{\langle x, v\rangle \mid x \in X\} \geqslant\langle y, v\rangle=f(v)$.

This holds for every $v \neq 0$. For $v=0$ this inequality is obvious, and we conclude that $\sigma(X) \geqslant f$, which was to be proved.

Remark 4.11. We can give an alternative proof of Proposition 4.10(b) which uses the upper slope transform for functions discussed in Section 3.4.

Assume that $f$ is 1.s.c. and sublinear, and that $f \not \equiv+\infty$. We show that $f=\sigma(X)$ for some (closed, convex) set $X$. Consider the function $g=\mathscr{Y}_{\vee}-(f)$. Since $f \not \equiv+\infty$ we have $g(x)<+\infty$ for all $x$. The sublinearity of $f^{\prime}$ in combination with Proposition 3.18(d)-(e) implies that $g(r x)=y(x)$ for $r>0, x \in \mathbb{R}^{d}$. We conclude therefore that $g$ assumes only the values $-\infty$ and 0 , and so $g=l_{\wedge}(X)$ for some closed convex set $X$ (note that $g$ is u.s.c. and concave by Proposition 3.16(b)). Since $f \in \operatorname{Ran}(\mathscr{K})$ by Proposition 3.16(a), we have

$f=\mathscr{S}_{\vee} \mathscr{S}_{\vee}^{-}(f)=\mathscr{S}_{\vee}(g)=\mathscr{S}_{\vee}\left(l_{\wedge}(X)\right)=\sigma(-X)$,

by (4.4). This concludes the proof.

Schneider [24, Theorem 1.7.1] claims that every sublinear function is the support function of some 
convex body. Apparently, he forgot to include the requirement that this function is l.s.c.

We have the following analogue of Corollary 3.17.

\section{Corollary 4.12 .}

(a) $\sigma^{-} \sigma$ is a closing on $\mathscr{P}\left(\mathbb{R}^{d}\right)$ with invariance domain the closed convex sets in $\mathbb{R}^{d}$, i.e.,

$\sigma^{\leftarrow} \sigma(X)=\beta_{\mathrm{c}} \beta_{\wedge}(X)=\overline{\mathrm{co}}(X)$.

(b) $\sigma \sigma^{-}$is an opening on Fun( $\left.\mathbb{R}^{d}\right)$ with invariance domain the l.s.c. sublinear functions, i.e.,

$\sigma \sigma^{\leftarrow}(f)=\alpha_{,} x_{\mathrm{sl}}(f)$.

Proof. (a) follows from the previous results.

To prove (b) we still have to show that $\alpha_{,} \alpha_{\mathrm{s}}$ is an opening with invariance domain Fun $/\left(\mathbb{R}^{d}\right) \cap$ $\operatorname{Fun}_{\mathrm{sl}}\left(\mathbb{R}^{d}\right)$, the l.s.c. sublinear functions. Exploiting Proposition 2.3(a), it remains to show that $\alpha_{\mathrm{s} 1} \alpha_{,} \alpha_{\mathrm{s} 1}=\alpha_{1} \alpha_{\mathrm{sl}}$. We use the fact that the first term $\alpha_{\mathrm{sl}}$ at the left-hand side may be replaced by $\alpha_{\vee}$ (because of Lemma 4.3). Now

$\alpha_{\vee} \alpha_{1} \alpha_{s !}=\alpha_{\vee} \alpha_{1} \alpha_{\vee} \alpha_{s !}=\alpha_{1} \alpha_{\vee} \alpha_{s !}=\alpha_{,} \alpha_{s !}$,

(cf. Corollary 3.23) and this concludes the proof.

Many results in the literature follow easily if one uses Propostion 4.10 and Corollary 4.12. For example, in Satz 12.4, Leichtweiss [15] shows that

$\sigma\left(\overline{\mathrm{CO}}\left(X_{1} \cup \cdots \cup X_{m}\right)\right)=\sigma\left(X_{1}\right) \vee \cdots \vee \sigma\left(X_{m}\right)$,

if $X_{1}, X_{2}, \ldots, X_{m}$ are compact and convex. From the fact that $\overline{\mathrm{co}}(\cdot)=\sigma^{-} \sigma$ (hence $\sigma(\overline{\mathrm{co}}(X))=\sigma \sigma^{\leftarrow}$ $\sigma(X)=\sigma(X))$ and that $\sigma$ is a dilation, we find that

$\sigma\left(\bigcup_{i \in I} X_{i}\right)=\sigma\left(\overline{\mathrm{co}}\left(\bigcup_{i \in I} X_{i}\right)\right)=\bigvee_{i \in I} \sigma\left(X_{i}\right)$

for a collection $X_{i}, i \in I$, of arbitrary subsets of $\mathbb{R}^{d}$.

If one applies Proposition 2.1(b) to the adjunction $\left(\sigma^{\leftarrow}, \sigma\right)$, one finds that

$$
\sigma\left(\bigcap_{i \in I} X_{i}\right)=\alpha, \alpha_{\vee}\left(\bigwedge_{i \in I} \sigma\left(X_{i}\right)\right)
$$

if $X_{i}$ is closed and convex for every $i \in I$; cf. [13, Theorem. V-3.3.3(iii)].
We list properties of $\sigma$ and its adjoint $\sigma^{\leftarrow}$. For a set $X \subseteq \mathbb{R}^{d}$ and a vector $h \in \mathbb{R}^{d}$ we define $X_{h}$ as the translate of $X$ along $h$, i.e., $X_{h}=\{x+h \mid x \in X\}$.

Proposition 4.13. (Properties of $\sigma$ ). For $X, Y \subseteq \mathbb{R}^{d}, h$ $\in \mathbb{R}^{d}$, and $r>0$ :
(a) $\sigma\left(X_{h}\right)=\sigma(X)_{[h]}$,
(b) $\sigma(r X)=r \sigma(X)$
(c) $\sigma(-X)=\sigma(X)(-\cdot)$,
(d) $\sigma(X \oplus Y)=\sigma(X)+\sigma(Y)$.

Proposition 4.14. (Properties of $\sigma^{-}$). For $f \in$ Fun $\left(\mathbb{R}^{d}\right)$, $h \in \mathbb{R}^{d}$, and $r>0$ :

(a) $\sigma^{-}\left(f_{\mid h]}\right)=\sigma^{-}(f)_{h}$,

(b) $\sigma^{-}(r f)=r \sigma^{-}(f)$,

(c) $\sigma^{-}(f(r \cdot))=r \sigma^{-}(f)$,

(d) $\sigma^{-}(f(-\cdot))=-\sigma^{-}(f)$.

We substitute $f=\sigma(X)$ and $g=\sigma(Y)$ in (4.2) and get $\alpha_{\vee}(\sigma(X) \wedge \sigma(Y))=\sigma(X) \square \sigma(Y)$.

Applying $\alpha$, at both sides and using (4.8) yields $\sigma(X \cap Y)=\alpha_{,}(\sigma(X) \square \sigma(Y))$.

In $[22$, Corollary $16.4 .1 ; 13$, p. 227] similar results have been obtained.

\subsection{Polar, gauge, and support function}

The polar $X^{\circ}$ of a set $X \subseteq \mathbb{R}^{d}$ is defined by $X^{\circ}=\left\{y \in \mathbb{R}^{d} \mid\langle x, y\rangle \leqslant 1\right.$ for all $\left.x \in X\right\}$.

We define the operator $\pi$ by $\pi(X)=X^{\circ}$.

Let $\mathscr{P}^{\prime}\left(\mathbb{R}^{d}\right)$ be the opposite of the complete Boolean lattice $\mathscr{P}\left(\mathbb{R}^{d}\right)$ (see Section 2.1).

\section{Proposition 4.15.}

(a) $(\pi, \pi)$ is an adjunction between $\mathscr{P}^{\prime}\left(\mathbb{R}^{d}\right)$ and $\mathscr{P}\left(\mathbb{R}^{d}\right)$, in particular

$$
\left(\bigcup_{i \in l} X_{i}\right)^{\circ}=\bigcap_{i \in I} X_{i}^{\circ}
$$

for every collection $X_{i} \subseteq \mathbb{R}^{d}, i \in I$. 
(b) $\operatorname{Ran}(\pi)$ consists of the closed convex sets which contain the origin.

Proof. (a) We must show that $Y \subseteq \pi(X) \Leftrightarrow X \subseteq \pi(Y)$, for $X, Y \subseteq \mathbb{R}^{d}$. Because of the symmetry of this assertion, it suffices to prove ' $\Rightarrow$ '. Therefore, assume that $Y \subseteq \pi(X)$. We show that $X \subseteq \pi(Y)$. Take $x \in X$; we must show that $x \in \pi(Y)$, i.e., that $\langle y, x\rangle \leqslant 1$ for $y \in Y$. Since $Y \subseteq \pi(X)$ we have that $\langle y, x\rangle \leqslant 1$ for $x \in X, y \in Y$. This proves the result.

(b) It is easy to show that a set in the range of $\pi$ is closed, convex, and contains the origin. To prove the converse we observe that $\operatorname{Ran}(\pi)$ is closed under intersection. Every closed convex set containing the origin is the intersection of closed half planes containing the origin. A straightforward computation shows that $\pi^{2}$ leaves such half planes invariant, which means in particular that they lie in $\operatorname{Ran}(\pi)$. Now the result follows.

Corollary 4.16. $\pi^{2}$ is a closing on $\mathscr{P}\left(\mathbb{R}^{d}\right)$ and $\pi^{2}(X)=\overline{\mathrm{co}}(X \cup\{0\})$, for every $X \subseteq \mathbb{R}^{d}$.

Proof. The theory on adjunctions summarized in Section 2.1 gives that $\pi^{2}$ is a closing. Furthermore, Proposition 4.15(b) yields that the invariance domain of $\pi^{2}$ consists of the closed convex sets which contain the origin. Therefore, $\pi^{2}(X)$ is the smallest closed convex set containing the origin which is larger than $X$. This means that $\pi^{2}(X)=\overline{\mathrm{co}}(X \cup\{0\})$.

A similar result (though only for convex sets) can be found in $[22$, p. 125].

In Example 4.2(c) we have introduced the gauge function of a convex set containing the origin. We extend this definition to arbitrary subsets of $\mathbb{R}^{d}$ and put

$\gamma(X)(x)=\inf \{r>0 \mid x \in r X\}$.

Thus, $\gamma$ is a mapping from $\mathscr{P}\left(\mathbb{R}^{d}\right)$ into Fun $\left(\mathbb{R}^{d}\right)$. Furthermore, we define $\gamma^{-}:$Fun $\left(\mathbb{R}^{d}\right) \rightarrow \mathscr{P}\left(\mathbb{R}^{d}\right)$ by

$\gamma^{\leftarrow}(f)=\left\{x \in \mathbb{R}^{d} \mid \forall r>0: f(r x) \leqslant r\right\}$.

\section{Proposition 4.17.}

(a) $\left(\gamma, \gamma^{-}\right)$is an adjunction between $\mathscr{P}^{\prime}\left(\mathbb{R}^{d}\right)$ and $\operatorname{Fun}\left(\mathbb{R}^{d}\right)$. (b) $\operatorname{Ran}(\gamma)$ consists of all positively homogeneous functions.

(c) $\operatorname{Ran}\left(\gamma^{-}\right)$consists of all sets $X \subseteq \mathbb{P}^{d}$ with the property that

$x \in X \Leftrightarrow \forall r \in(0,1): r x \in X$.

Proof. (a) We must show that $X \subseteq \eta^{-}(f) \Leftrightarrow f \leqslant$ $i(X)$.

' $\Rightarrow$ ': Assume $X \subseteq \eta^{-}(f)$; we show that $f^{\prime} \leqslant i^{\prime}(X)$. Suppose that, for some $x, f(x)>\inf \{r>0 \mid x \in r X\}$. Then there is an $r<f^{\prime}(x)$ such that $x \in r X$, i.e., $(1 / r) x \in X$. Since $X \subseteq r^{-}(f)$, this means that $(1 / r) x \in r^{-}(f)$. Then $f((s / r) x) \leqslant s$ for every $s>0$. Substituting $s=r$ yields that $f^{\prime}(x) \leqslant r$, a contradiction. ' $\Leftarrow$ ': Assume that $f \leqslant i(X)$; we show that $X \subseteq \eta^{-}(f)$. Suppose $x \in X$ and $r>0$. Then

$f(r x) \leqslant \gamma(X)(r x)=\inf \{s>0 \mid r x \in s X\} \leqslant r$.

This yields that $x \in 7^{-}(f)$.

(b) It is easy to see that every function $;(X)$ is positively homogeneous. We have to show that for every positively homogeneous function $f$ there exists a set $X$ such that $;(X)=f$. Define $X=\gamma^{-}(f)=\{x \mid \forall r>0: f(r x) \leqslant r\}$. We show that $\gamma(X)=f$. Since $n$ is a closing, it follows immediately that $\gamma(X)=\cdots-(f) \geqslant f$. Thus, it remains to be shown that $\gamma(X) \leqslant f$. Assume that $\gamma(X)(y)>f(y)$ for some $y$. Choose $r$ such that $i^{\prime}(X)(y)>r>f(y)$. Then $\gamma(X)(y)=\inf \{s>0 \mid y \in s X\}>r$, meaning that $y \notin r X$. This yields that $(1 / r) y \notin X=\{x \mid \forall s>0: f(s x)$ $\leqslant s\}$. Hence there exists an $s>0$ such that $f(s \cdot(1 / r) y)>s$. As $f$ is positively homogeneous this means that

$s<f\left(s \cdot \frac{1}{r} y\right)=\frac{s}{r} f(y)<\frac{s}{r} \cdot r=s$,

a contradiction.

(c) It is rather easy to show that for every set $X=\gamma^{-}(f)$ property (4.9) holds. To prove the converse, assume that $X \neq \emptyset$ is a set for which (4.9) holds. Define $f=\eta^{\prime}(X)$ and $X^{\prime}=\eta^{-}(f)$. We show that $X^{\prime}=X$. The composition $\gamma^{-} \gamma$ is an opening on $\mathscr{P}^{\prime}\left(\mathbb{R}^{d}\right)$, hence a closing on $\mathscr{P}\left(\mathbb{R}^{d}\right)$. This yields that $X \subseteq \gamma^{-} \gamma(X)=X^{\prime}$. Therefore, we must show that $X^{\prime} \subseteq X$. Suppose that $y \in X^{\prime}$, that is, $f(r y) \leqslant r$ for every $r>0$. This means in particular that $f(y) \leqslant 1$. As $f=\gamma(X)$, this implies that inf $\{s>0 \mid y \in s X\} \leqslant 1$. 
If $y=0$ then $y \in X$. Therefore, we may assume that $y \neq 0$. We distinguish between two cases.

1. $y \in s X$ for some $0<s \leqslant 1$. Then $(1 / s) y \in X$, and (4.9) yields that $y \in X$.

2. $y \in s_{n} X$ for some sequence $\left\{s_{n}\right\}$ converging to 1 from above. This yields that $\left(1 / s_{n}\right) y \in X$. Now (4.9) implies that $r y \in X$ for $r<1$, hence $y \in X$.

In particular, Proposition 4.17(c) gives that a set which lies in the range of $i^{-}$and which contains the origin, is star-shaped with respect to the origin, i.e., if $x \in X$ then $r x \in X$ for $0 \leqslant r \leqslant 1$.

From Proposition 4.17(a) we know that $\gamma^{t} \gamma$ is a closing on $\mathscr{P}\left(\mathbb{R}^{d}\right)$ and that $\varkappa^{-}$is a closing on Fun $\left(\mathbb{R}^{d}\right)$. We derive explicit expressions for these operators.

Let $\omega: \mathscr{P}\left(\mathbb{R}^{d}\right) \rightarrow \mathscr{P}\left(\mathbb{R}^{d}\right)$ be given by

$\omega(X)=\bigcap_{s>1} \bigcup_{0<r \leqslant s} r X$,

and let $\Gamma:$ Fun $\left(\mathbb{R}^{d}\right) \rightarrow$ Fun $\left(\mathbb{R}^{d}\right)$ be given by

$\Gamma(f)(x)=\bigvee_{r>0} \frac{1}{r} f(r x)$.

\section{Proposition 4.18.}

(a) $\gamma^{-} \gamma=\omega$, and this operator defines a closing on $\mathscr{P}\left(\mathbb{R}^{d}\right)$.

(b) $\gamma \gamma^{\leftarrow}=\Gamma$, and this operator defines a closing on $\operatorname{Fun}\left(\mathbb{R}^{d}\right)$

Proof. (a) A straightforward computation shows that

$$
\begin{aligned}
\gamma^{-} \gamma(X)= & \left\{x \in \mathbb{R}^{d} \mid \forall r>0: \gamma(X)(r x) \leqslant r\right\} \\
= & \left\{x \in \mathbb{R}^{d} \mid \forall r>0:\right. \\
& \quad \inf \{s>0 \mid r x \in s X\} \leqslant r\} \\
= & \left\{x \in \mathbb{R}^{d} \mid \forall r>0:\right. \\
& r \cdot \inf \{s>0 \mid x \in s X\} \leqslant r\} \\
= & \left\{x \in \mathbb{R}^{d} \mid \inf \{s>0 \mid x \in s X\} \leqslant 1\right\} .
\end{aligned}
$$

Therefore, $x \in \gamma^{\leftarrow} \gamma(X)$ iff inf $\{s>0 \mid x \in s X\} \leqslant 1$. We show that $\gamma^{\leftarrow} \gamma(X)=\omega(X)$.

To prove ' $\subseteq$ ' assume that $x \in \gamma^{\leftarrow} \gamma(X)$, that is, $\inf \{s>0 \mid x \in s X\} \leqslant 1$. There are two possibilities:

1. $x \in s X$ for some $0<s \leqslant 1$. In this case it is obvious that $x \in \omega(X)$.
2. $x \in s_{n} X$ for some sequence $\left\{s_{n}\right\}$ converging to 1 from above. Then $x \in \bigcup_{0<r \leqslant s} r X$, for every $s>1$, and it follows that $x \in \omega(X)$.

To prove ' $\supseteq$ ' assume that $x \in \omega(X)$. This implies that $\inf \{r>0 \mid x \in r X\} \leqslant s$ for every $s>1$. But then inf $\{r>0 \mid x \in r X\} \leqslant 1$, yielding that $x \in \gamma^{-} \gamma(X)$.

(b) For $\gamma^{+}$we derive

$$
\begin{aligned}
\gamma \gamma^{-}(f) & =\inf \left\{r>0 \mid x \in r \gamma^{\leftarrow}(f)\right\} \\
& =\inf \{r>0 \mid x \in r\{x \mid \forall s>0: f(s x) \leqslant s\}\} \\
& =\inf \left\{r>0 \mid \forall s>0: f\left(\frac{s}{r} x\right) \leqslant s\right\} \\
& =\inf \{r>0 \mid \forall s>0: f(s x) \leqslant r s\} \\
& =\inf \left\{r>0 \mid \forall s>0: \frac{1}{s} f(s x) \leqslant r\right\} \\
& =\inf \left\{r>0 \mid \bigvee_{s>0} \frac{1}{s} f(s x) \leqslant r\right\} \\
& =\bigvee_{s>0} \frac{1}{s} f(s x) \\
& =\Gamma(f)(x) .
\end{aligned}
$$

This proves the result.

From the literature on convex sets [22, Thm.14.5] it is well-known that

$\gamma\left(X^{\circ}\right)=\sigma(X)$

if $X$ is closed, convex, and if $0 \in X$. Since $\pi^{2}(X)$ satisfies these constraints for every $X \subseteq \mathbb{R}^{d}$ (cf. Corollary 4.16) we get that $\gamma \pi^{3}=\sigma \pi^{2}$. But $\pi^{3}=\pi$ since $(\pi, \pi)$ is an adjunction, hence $\gamma \pi=\sigma \pi^{2}$. This yields that $\gamma \pi^{2}=\sigma \pi^{3}=\sigma \pi$.

Proposition 4.19. $\gamma \pi=\sigma \pi^{2}$ and $\gamma \pi^{2}=\sigma \pi$.

\section{Two applications}

In this section we outline the applicability of slope transforms for two different problems in nonlinear image analysis, distance computation and partial differential equations of the evolution type that model morphological scale space. 


\subsection{Distance transforms}

Let $\|\cdot\|_{p}$, denote the norm on $\mathbb{R}^{d}$ given by

$\|x\|_{p}=\left(\left|x_{1}\right|^{p}+\left|x_{2}\right|^{p}+\cdots+\left|x_{d}\right|^{p}\right)^{1 / p}$.

Given a set $X \subseteq \mathbb{R}^{d}$, we define its distance transform (also known as its distance function) with respect to $p$-norm by

$D_{p}(X)(x)=\bigwedge_{1, x}\|x-y\|_{p}$

The distance transform has various applications in image analysis and computer vision. For example, its thresholds at levels $r>0$ yield the multiscale dilations of $X$ by the balls $r B_{p}$, where $B_{p}$ is the unit ball with respect to the $p$-norm. Further (for $p=2$ ), its local maxima provide the points of the skeleton (medial) axis of $X^{\prime}$. Then, if we consider the upper indicator function $l(X)$, and the convex conical structuring function

$y(x)=\|x\|_{l,}$,

it follows that

$$
\begin{aligned}
D_{p}(X)(x) & =\bigwedge_{y}\left(I_{y}(X)(y)+\|x-y\|_{p}\right) \\
& =(I(X) \sqcap g)(x) ;
\end{aligned}
$$

see also |13, Example 2.3.5]. In other words, the distance transform of $X$ can be obtained as the infimal convolution of the upper indicator function of $X$ with the conical norm function. This infimal convolution is equivalent to passing the input signal, i.e., the set's upper indicator function $I_{\vee}(X)$, through an ETI system with slope response

$y^{\prime}(r)=\bigwedge_{r, \| v^{\prime \prime}}\|x\|_{p}-\langle x, v\rangle$

It is evident that $g^{\wedge}(v) \leqslant 0$. Furthermore, by using Hölder's inequality we get

$\| x, r\rangle \mid<\|x\|_{n} \cdot\|v\|_{q}$,

where the exponent $q$ is determined by

$$
\frac{1}{p}+\frac{1}{q}=1
$$

Thus, we find that

$g^{\wedge}(v) \geqslant \bigwedge_{x \in \mathbb{R}^{\prime \prime}}\|x\|_{p}\left(1-\|v\|_{q}\right)$.

Therefore, $g^{\wedge}$ is equal to

$g^{\wedge}(v)= \begin{cases}0, & \|v\|_{q} \leqslant 1 \\ -\infty, & \|v\|_{q}>1\end{cases}$

That is, the distance transform is the output of an idealcutoff slope-selective filter that rejects all input planes whose slope vector falls outside the unit ball with respect to the $\|\cdot\|_{q}$ norm, and passes all the others unchanged.

\subsection{Hamilton-Jacobi equations for multiscale morphology}

Let $K: \mathbb{R}^{d} \rightarrow \mathbb{R}$ be a u.s.c. concave function. Consider the parameterized family $\left\{K_{t} \mid t \geqslant 0\right\}$ given by

$$
\begin{cases}K_{0}=q \wedge & \text { (i.e., } K_{0}(x)=0 \text { if } x=0 \\ & \text { and }-\infty \text { elsewhere), } \\ K_{t}(x)=t K(x / t), & x \in \mathbb{R}^{d}, t>0 .\end{cases}
$$

This family satisfies the semigroup property

$K_{l}, K_{s}=K_{t+s}, \quad s, t \geqslant 0$.

Let, for a given input $f$, the function $u:[0, \infty) \times$ $\mathbb{R}^{d} \rightarrow \mathbb{R}^{d}$ be given by

$u(t, x)=\left(f f, K_{t}\right)(x)$.

We have the following heuristic derivation of a PDE (partial differential equation) for $u(t, x)$. First, we note that

$$
\frac{\partial u}{\partial t}(t, x)=\lim _{s ! 0} \frac{1}{s}[u(t+s, x)-u(t, x)] .
$$

We use that $u(t+s, \cdot)=u(t, \cdot) \oplus K_{s}$;

$$
\frac{\partial u}{\partial t}(t, x)=\lim _{s ! 0} \frac{1}{s}\left[\bigvee_{h \in \mathbb{R} d}(u(t, x-h)\right.
$$

$$
+s K(h / s)-u(t, x))]
$$




$$
\begin{aligned}
=\lim _{s ! 0} \frac{1}{s} & {\left[\bigvee_{h \in \mathbb{R}^{\prime}}(u(t, x-s h)\right.} \\
& -u(t, x)+s K(h))] .
\end{aligned}
$$

Presuming that $u(t, x)$ is differentiable in $x$ we may write

$u(t, x-s h)-u(t, x)=-\langle s h, \nabla u(t, x)\rangle+o(s), \quad s \downarrow 0$, where $s^{-1} o(s) \rightarrow 0$ as $s \downarrow 0$. Here $\nabla u$ denotes the gradient of $u$. We get

$$
\begin{aligned}
\frac{\partial u}{\partial t}(t, x) & =\lim _{s \mid 0} \bigvee_{h \in \mathbb{R} \cdot t}[K(h)-\langle h, \nabla u(t, x)\rangle+o(s)] \\
& =\bigvee_{h \in \mathbb{R}^{\prime} \cdot}[K(h)-\langle h, \nabla u(t, x)\rangle] \\
& =K^{\vee}(\nabla u(t, x)),
\end{aligned}
$$

where $K^{\vee}$ is the upper slope transform of $K$. Writing $u_{t}=\partial u / \partial t$ we arrive at the evolution equation:

$u_{t}=K^{\vee}(\nabla u)$.

Before we solve this equation, we give a short treatment of a class of PDEs known from mathematical physics, the so-called Hamilton-Jacobi equation, given by

$w_{t}+H(\nabla w)=0$,

where the Hamiltonian $H: \mathbb{R}^{d} \rightarrow \mathbb{R}$ is a convex function which satisfies

$\lim _{\|p\| \rightarrow \infty} \frac{H(p)}{\|p\|}=+\infty$.

(A function which satisfies (5.4) is called coercive.) The Young-Fenchel conjugate $L=H^{\star}$, called the Lagrangian, is finitely-valued, convex, and coercive. If $f$ is bounded and 1.s.c., then the function $w(t, \cdot)$ given by

$w(t, \cdot)= \begin{cases}f, & t=0, \\ f \square L_{t}, & t>0,\end{cases}$

where $L_{t}(x)=t L(x / t)$, has the following properties:

- $w(t, x) \rightarrow f(x)$ as $t \downarrow 0$, for every $x$;

- $w$ is locally Lipschitz continuous (hence differentiable almost everywhere);
- at every $(t, x)$ where $w$ is differentiable, it satisfies the Hamilton-Jacobi equation (5.3).

In the literature $w$ is called the viscosity solution of the Hamilton--Jacobi equation; see [16] for a comprehensive account. For other literature on the Hamilton-Jacobi equation, see [3].

The relation between the Hamilton-Jacobi equation (5.3) and our morphological evolution equation (5.2) is as follows: if we substitute $u=-w$ in (5.2) we get

$-w_{t}=K^{\vee}(-\nabla w)$.

With relation $(3.10)$ this can be written as

$w_{t}+(-K)^{\star}(\nabla w)=0$.

If we assume that $K$ is concave, u.s.c., and coercive, then the Hamiltonian $H=(-K)^{\star}$ is convex, l.s.c., and coercive, and the Lagrangian is given by

$L=H^{\star}=(-K)^{\star}=-K$.

The viscosity solution of $(5.5)$ with $w(0)=-u(0)=$ - $f$ is given by

$w(t, \cdot)=(-f) \square L_{t}=(-f) \square(-K)_{t}$.

Therefore, the 'viscosity solution' of our morphological evolution equation (5.2) is given by

$u(t, \cdot)=-(-f) \square(-K)_{t}=f \oplus K_{t}$.

We summarize our findings in the following result.

Proposition 5.1. Assume that the function $K$ is concave, u.s.c., and coercive. If $f$ is bounded and u.s.c. then the function $u(t, \cdot)=f \oplus K_{t}$ satisfies

- $u(t, x) \rightarrow f(x)$ as $t \downarrow 0$, for every $x$;

- $u$ is locally Lipschitz continuous (hence differentiable almost everywhere);

- at every $(t, x)$ where $u$ is differentiable, it satisfies the evolution equation (5.2).

For example, if $K(x)=-\frac{1}{2}\|x\|^{2}$, then $K^{\vee}(v)=\frac{1}{2}\|v\|^{2}$. For some related results on multiscale morphological evolution equations, the reader may refer to $[1,20,29]$.

If we take a flat structuring element, $K=\imath_{\wedge} A$ with $A$ a closed convex set, we arrive at the equation $u_{t}=\sigma(-A)(\nabla u)$. 
If $A$ is symmetric $(A=-A)$ and contains the origin in its interior, then $\sigma(-A)=\sigma(A)$ equals the gauge functional of the polar set

$A^{\circ}=\{x \mid\langle a, x\rangle \leqslant 1$, for all $a \in A\}$.

This gauge function is a norm with unit ball $A^{\circ}$. The examples treated in $[5,6]$ fall inside this class.

\section{Conclusions and discussion}

In recent studies in mathematical morphology $[17,18,7]$, the slope transform has emerged as a transform which has similar properties with respect to morphological signal processing as the Fourier transform does with respect to linear signal processing. Its main property is that it transforms a supremal convolution (morphological dilation) into an addition, in the very same way as the Fourier transform transforms a linear convolution into a multiplication. At an earlier stage, Ghosh [9] built a computational framework for Minkowski addition and subtraction of convex and non-convex polygons based on the so-called slope diagram, a concept which is very closely related to the slope transform.

There is, however, an important difference between the Fourier transform and its morphological counterpart, the slope transform. The Fourier transform is invertible, whereas the slope transform only has an adjoint in the sense of adjunctions. This means that the 'inverse' of the slope-transformed signal is not the original signal but only an approximation within the subcollection of convex or concave signals. This is why convex analysis plays such a prominent role in the study of the slope transform. We have pointed out various relations with known concepts from the theory of convex sets and functions, such as the Legendre transform, the (Young-Fenchel) conjugate, the support function, the gauge function, and set polarity. In particular, we have explained how such classical concepts can be studied as complete lattice operators. This allows one to reformulate various known results, and even to extend a number of them.

The complete lattices considered in this papers are either lattices of sets or of functions. For an abstract treatment of the slope transform on arbitrary complete lattices we refer to [12].

\section{References}

[1] L. Alvarez, J. Morel, Formalization and computational aspects of image analysis, Acta Numerica (1994) 1-59.

[2] R. Bellman, W. Karush, On the maximum transform, J. Math. Anal. Appl. 6 (1963) 67-74.

[3] S.H. Benton, Jr., The Hamilton-Jacobi Equation: A Global Approach, Academic Press. New York, 1977.

[4] G. Birkhoff, Lattice theory, 3rd ed., American Mathematical Society Colloquium Publications, Vol. 25, American Mathematical Society, Providence, RI, 1967.

[5] R.W. Brockett, P. Maragos, Evolution equations for continuous-scale morphology, in: Proc. IEEE Internat. Conf. Acoust. Speech Signal Process., San Fransisco, CA, 1992. pp. III: $125-128$.

[6] R.W. Brockett, P. Maragos, Evolution equations for continuous-scale morphological filtering, IEEE Trans. Signal Process. 42 ( 1994 ) 3377-3386.

[7] L. Dorst, R. Van den Boomgaard, Morphological signal processing and the slope transform, Signal Processing 38 (1994) $79-98$

[8] W. Fenchel, On conjugate convex functions, Canad. J. Math. 1 (1949) 73-77.

[9] P.K. Ghosh, A unified computational framework for Minkowski operations, Comput. Graphics 17 (1993) 357-378.

[10] H.J.A.M. Heijmans, A note on the umbra transform in grayscale morphology, Pattern Recognition Letters 14 (1993) $877-881$.

[11] H.J.A.M. Heijmans, Morphological Image Operators, Academic Press, Boston, 1994.

[12] H.J.A.M. Heijmans, I.S. Molchanov, Morphology on complete lattices: linearity, convexity and the slope transform, J. Math. Imaging Vision (1997).

[13] J.-B. Hiriart-Umuty, C. Lemaréchal, Convex Analysis and Minimization Algorithms 1, Springer, Berlin, 1993.

[14] J.-B. Hiriart-Urruty, C. Lemaréchal, Convex Analysis and Minimization Algorithms II, Springer, Berlin, 1993.

[15] K. Leichtweiss, Konvexe Mengen, Springer, Berlin, 1980.

[16] P.L. Lions, Generalized Solutions of Hamilton-Jacobi Equations, Pitman, Boston, 1982.

[17] P. Maragos, Morphological systems: Slope transforms and $\max -\mathrm{min}$ difference and differential equations, Signal Processing 38 (1994) 57-77.

[18] P. Maragos, Slope transforms: theory and application to nonlinear signal processing, IEEE Trans. Signal Process. 43 (1995) $864-877$.

[19] G. Matheron, Random Sets and Integral Geometry, Wiley, New York, 1975.

[20] J. Mattioli, Differential relations of morphological operators, in: Proc. Ist International Workshop on Mathematical Morphology and its Application to Signal Processing, Barcelona, Spain, 1993, pp. 162-167.

[21] A.W. Roberts, D.E. Varberg, Convex Functions, Academic Press, New York, 1973.

[22] R.T. Rockafellar, Convex Analysis, Princeton University Press, Princeton, 1972. 
[23] C. Ronse, P. Moreau, Generation of shading-off in images by extrapolation of Lipschitz functions, Graphical Models Image Process. 58 (4) (1996) 314-333.

[24] R. Schneider, Convex Bodies: the Brunn-Minkowski Theory, Cambridge University Press, Cambridge, 1993.

[25] J. Serra, Image Analysis and Mathematical Morphology, Academic Press, London, 1982.

[26] J. Serra, Ed. Image Analysis and Mathematical Morphology. II: Theoretical Advances, Academic Press, London, 1988.

[27] J. Serra, A sampling approach based on equicontinuity, in: J. Serra, P. Soille (Eds.), Mathematical Morphology and its Applications to Image Processing, Kluwer Academic Publishers, Dordrecht, 1994, pp. 117-124.

[28] F.A. Valentine, Convex Sets, McGraw-Hill, New York, 1964.

[29] R. van den Boomgaard, A. Smeulders, The morphological structure of images: the differential equations of morphological scale space, IEEE Trans. Pattern Anal. Machine Intelligence 16 (1994) 1101-1113.

[30] J. van Tiel, Convex Analysis, Wiley, New York, 1984. 